\title{
Robust output regulation of the linearized Boussinesq equations with boundary control and observation
}

\author{
Konsta Huhtala ${ }^{1}$ D $\cdot$ Lassi Paunonen $^{1} \cdot$ Weiwei $\mathrm{Hu}^{2}$
}

Received: 11 January 2021 / Accepted: 29 October 2021 / Published online: 8 December 2021

(C) The Author(s) 2021

\begin{abstract}
We study a temperature and velocity output tracking problem for a two-dimensional room model with the fluid dynamics governed by the linearized translated Boussinesq equations. Additionally, the room model includes finite-dimensional models for actuation and sensing dynamics; thus, the complete model dynamics are governed by an ODE-PDE-ODE cascade. As the main contribution, we design a low-dimensional internal model-based controller for robust output tracking of the room model. The controller's performance is demonstrated through a numerical example.
\end{abstract}

Keywords Partial differential equations · Output regulation · Linear systems · Fluid flows · Coupled systems

\section{Introduction}

We consider fluid temperature and velocity control for a two-dimensional room model. In the model, behavior of the fluid within the room is described by the linearized Boussinesq equations. The Boussinesq equations are a system of partial differential equations coupling the fluid flow dynamics given by the incompressible Navier-Stokes

\footnotetext{
The research was supported by the Academy of Finland Grant No. 310489 held by L. Paunonen. L. Paunonen was funded by the Academy of Finland Grant No. 298182. W. Hu was partially supported by the NSF Grant DMS-1813570.

Konsta Huhtala

konsta.huhtala@tuni.fi

Lassi Paunonen

lassi.paunonen@tuni.fi

Weiwei Hu

Weiwei.Hu@uga.edu

1 Faculty of Information Technology and Communication Sciences, Tampere University, PO. Box 692, 33101 Tampere, Finland

2 Department of Mathematics, University of Georgia, Athens, GA 30602, USA
} 


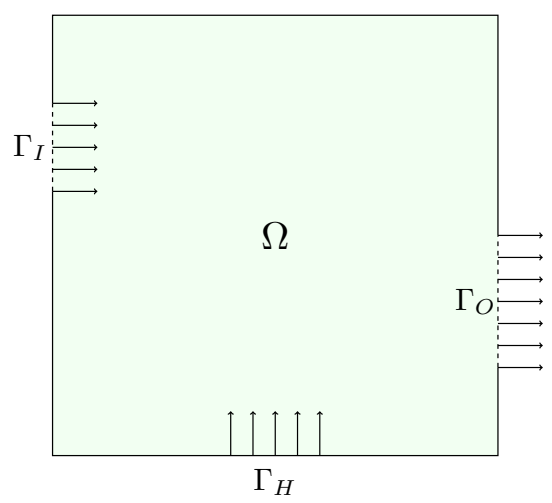

Fig. 1 A room with the boundary regions of interest highlighted

equations with the fluid temperature dynamics governed by the advection-diffusion equation, and they are commonly used for modeling non-isothermal flows, see, e.g., $[1,10,12]$. In this paper, we consider the linearized Boussinesq equations. As the main control problem, we study output tracking for the room model, where a mix of observations on the fluid temperature and velocity must converge to a desired reference trajectory over time, i.e.,

$$
\left\|y(t)-y_{\mathrm{ref}}(t)\right\| \rightarrow 0 \text { as } t \rightarrow \infty
$$

where $y(t) \in \mathbb{R}^{p_{Y}}$ is the observation and $y_{\mathrm{ref}}(t) \in \mathbb{R}^{p_{Y}}$ is the reference output. The considered reference outputs are of the form

$$
y_{\mathrm{ref}}(t)=a_{0}(t)+\sum_{i=1}^{q_{s}} a_{i}(t) \cos \left(\omega_{i} t\right)+b_{i}(t) \sin \left(\omega_{i} t\right),
$$

where $0=\omega_{0}<\omega_{1}<\cdots<\omega_{q_{s}}$ are known frequencies and $a_{i}(t), b_{i}(t) \in \mathbb{R}^{p_{Y}}$ are polynomial vectors with possibly unknown coefficients but known maximal degrees. As the main contribution of this paper, we design a finite-dimensional controller for output tracking of the room model with the room geometry depicted in Fig. 1.

Fluid dynamics within the room are governed by the linearized translated Boussinesq equations. We focus on a control setup typical for rooms, where the physical control inputs act on the fluid near some parts of the boundary of the room, i.e., the walls, the floor or the roof, cf. [10,12]. In the model, the fluid flows into and out of the room through the boundary regions $\Gamma_{I}$ and $\Gamma_{O}$, which represent an inlet and an outlet, respectively. Both the fluid velocity and the fluid temperature are controlled within $\Gamma_{I}$, cf. [12]. Additionally, the fluid temperature is controlled within $\Gamma_{H}$ by a radiating heater, but no velocity control is applied within $\Gamma_{H}$ and there is no fluid flow through this boundary section. Observations on the fluid are performed both within the boundary regions and inside the spatial domain. In addition to the fluid dynamics, the room model includes finite-dimensional dynamical models for the actuators and the sensors related to the fluid control and observation, respectively. Dynamic actuator 
modeling has been reported to increase model accuracy for an acoustic model in [46], and has been argued to be a more realistic approach to system modeling in general [13]. The complete room model is thus a coupled ODE-PDE-ODE model. Compared to a model with PDE dynamics only, the room model is more complex in the sense of having extended dynamics, but the control and observation operations are bounded. From now on, we will refer to the full ODE-PDE-ODE room model as the cascade system.

We achieve the output convergence (1) for the room model by implementing a controller introduced in [34]. The controller is based on the internal model principle, see $[17,19,35]$, and has several desirable properties. It can be used in control of unstable systems, which is essential for this paper due to the fact that the linearized Boussinesq equations may be unstable [12] (depending on the room geometry and physical parameters). The controller does not require complete state information of the system but rather only uses the observation $y(t)$, and since the controller is based on a finitedimensional approximation of the room model combined with model reduction, it is of low-order for fast computations. Finally, the controller is robust in that it tolerates small system uncertainties and rejects disturbance signals of the form

$$
u_{d}(t)=c_{0}(t)+\sum_{i=1}^{q_{s}} c_{i}(t) \cos \left(\omega_{i} t\right)+d_{i}(t) \sin \left(\omega_{i} t\right)
$$

which can be applied either within the boundary or inside the spatial domain of the room. Here, $\omega_{i}$ are the same frequencies as in (2) and $c_{i}(t), d_{i}(t) \in \mathbb{R}^{d}$ are polynomial vectors with possibly unknown coefficients but known maximal degrees. For linear systems, also several alternative output tracking controllers have been designed. However, these control solutions typically lack the robustness property of fault tolerance and disturbance rejection, cf. [14,18,45], are designed for stable systems only, cf. [22,38], or are infinite-dimensional, cf. [23,33].

Most of the previous work regarding control of the Boussinesq equations focuses on stabilization $[12,26,37,42]$. Examples of output tracking for both nonlinear and linear thermal fluid flows based on state feedback have been considered in [1] and references therein, and solution methods for the related regulator equations have been further developed in [2,3]. Additionally, robust output tracking for a simplified room model with only temperature dynamics and in-domain control and observation has been studied in [27]. Finally, addition of the actuator dynamics for classes of linear systems has previously been considered in [11,13,31].

In this work, we utilize the concept of abstract boundary control systems, see [16, Ch. 3.3], [15], [40, Ch. 10], to formulate the abstract system presentation for the cascade system with temperature, velocity and ODE dynamics. The boundary control system framework appears to not have been used in the analysis of incompressible Navier-Stokes-type fluid flows previously, yet it is a natural presentation choice for systems with boundary inputs and can be translated to the more familiar abstract state space formulation, see [40, Ch. 10]. We also use the boundary control system framework to study effects of the additional ODE dynamics on exponential stabilizability and exponential detectability of the room model. Furthermore, the boundary control 
system formulation could be used to justify alternative controller designs, namely those introduced in [28].

The paper is organized as follows. In Sect. 2, we first present the complete room model. We formulate the cascade system as an abstract linear control system in Sect. 2.2. Section 2.3 is devoted for stabilizability and detectability analysis of the cascade system in terms of us presenting sufficient conditions for these properties. In Sect. 3, we couple the room model with an error feedback controller to guarantee the output convergence (1). The controller is based on [34] and we present the design process for the cascade system. In Sect. 4, we present a numerical example of robust output tracking for the boundary controlled linearized Boussinesq equations with a mix of boundary and in-domain observations and including actuator and sensor dynamics. Finally, the paper is concluded in Sect. 5.

We use the following notation. For a linear operator $A, D(A), \mathcal{R}(A)$ and $\mathcal{N}(A)$ denote its domain, range and kernel, respectively. The spectrum of $A$ is denoted by $\sigma(A)$ and the resolvent set by $\rho(A)$. The set of bounded linear operators from $X$ to $Y$ is denoted by $\mathcal{L}(X, Y)$. Finally, $\langle\cdot, \cdot\rangle_{\Omega}$ and $\langle\cdot, \cdot\rangle_{\Gamma}$ denote the $L^{2}$-inner product or duality pairing on the two-dimensional domain $\Omega$ and on the one-dimensional domain $\Gamma$, respectively.

\section{The room model}

We consider a two-dimensional model of a room depicted in Fig. 1 with the interior $\Omega$ and the boundary $\Gamma$. The room has two disjoint vents; an inlet $\Gamma_{I}$ and an outlet $\Gamma_{O}$. We denote the walls of the room by $\Gamma_{W}=\Gamma \backslash\left(\Gamma_{I} \cup \Gamma_{O}\right)$ and assume "no-slip" velocity condition at the walls. Regarding temperature, we assume that there is a radiative heater located within $\Gamma_{H} \subset \Gamma_{W}$ and on $\Gamma_{W} \backslash \Gamma_{H}$ the temperature is fixed. In addition to the radiator, the fluid flow and the fluid temperature within the room are affected by Robin boundary control within the inlet. Finally, the fluid is assumed to be stress-free with unforced heat flux within the outlet.

We next formulate the linearized Boussinesq equations around a steady-state solution of the Boussinesq equations. The linearized Boussinesq equations are used to describe the flow and temperature evolution of the fluid within the room. The system of PDEs is coupled with abstract ODE systems governing the actuation and sensing dynamics, and we present the cascade system in an abstract form. Finally, we consider stabilizability and detectability properties of the cascade system

\subsection{The linearized translated Boussinesq equations with actuation and sensing}

The Boussinesq equations for $\xi \in \Omega$ and $t \geq 0$ are given by

$$
\begin{aligned}
\dot{w}(\xi, t)= & \frac{1}{\operatorname{Re}} \Delta w(\xi, t)-(w(\xi, t) \cdot \nabla) w(\xi, t)-\nabla q(\xi, t) \\
& +\hat{e}_{2} \frac{G r}{\operatorname{Re}^{2}} T(\xi, t)+f_{w}(\xi)
\end{aligned}
$$




$$
\begin{aligned}
\dot{T}(\xi, t) & =\frac{1}{\operatorname{RePr}} \Delta T(\xi, t)-w(\xi, t) \cdot \nabla T(\xi, t)+f_{T}(\xi), \\
0 & =\nabla \cdot w(\xi, t), \quad w(\xi, 0)=w_{0}(\xi), \quad T(\xi, 0)=T_{0}(\xi),
\end{aligned}
$$

where $T$ is the temperature, $q$ is the pressure, and $w=\left[w_{1}, w_{2}\right]^{\mathrm{T}}$ is the velocity of the fluid. The functions $f_{w}$ and $f_{T}$ represent a body force and a heat source, respectively, and $\hat{e}_{2}=[0,1]^{\mathrm{T}}$ indicates the direction of buoyancy. Finally, the condition $0=$ $\nabla \cdot w(\xi, t)$ describes incompressibility of the fluid, $R e$ is the Reynolds number, $G r$ is the Grashof number and $P r$ is the Prandtl number. By linearizing the above equations around a steady-state solution $\left(w_{s s}, q_{s s}, T_{s s}\right)$ of (4) using the change of variables $v(\xi, t)=w(\xi, t)-w_{s s}(\xi), \theta(\xi, t)=T(\xi, t)-T_{s s}(\xi), p(\xi, t)=q(\xi, t)-q_{s s}(\xi)$, we arrive at the linearized translated Boussinesq equations

$$
\begin{aligned}
& \dot{v}(\xi, t)=\frac{1}{\operatorname{Re}} \Delta v(\xi, t)-\left(w_{s s}(\xi) \cdot \nabla\right) v(\xi, t)-(v(\xi, t) \cdot \nabla) w_{s S}(\xi) \\
& -\nabla p(\xi, t)+\hat{e}_{2} \frac{G r}{R e^{2}} \theta(\xi, t), \\
& \dot{\theta}(\xi, t)=\frac{1}{\operatorname{RePr}} \Delta \theta(\xi, t)-w_{s s}(\xi) \cdot \nabla \theta(\xi, t)-v(\xi, t) \cdot \nabla T_{s s}(\xi), \\
& 0=\nabla \cdot v(\xi, t), \quad v(\xi, 0)=v_{0}(\xi), \quad \theta(\xi, 0)=\theta_{0}(\xi) .
\end{aligned}
$$

We consider the linearized translated Boussinesq equations subject to the boundary conditions

$$
\begin{aligned}
& \left.\left(\mathcal{T}(v(\xi, t), p(\xi, t)) \cdot n+\alpha_{v} v(\xi, t)\right)\right|_{\Gamma_{I}}=\left[b_{v_{I}}(\xi) b_{d v_{I}}(\xi)\right]\left[\begin{array}{l}
u_{b v_{I}}(t) \\
u_{d v_{I}}(t)
\end{array}\right], \\
& \left.\left(\frac{1}{\operatorname{RePr}} \frac{\partial \theta}{\partial n}(\xi, t)+\alpha_{\theta} \theta(\xi, t)\right)\right|_{\Gamma_{I}}=\left[b_{\theta_{I}}(\xi) b_{d \theta_{I}}(\xi)\right]\left[\begin{array}{l}
u_{b \theta_{I}}(t) \\
u_{d \theta_{I}}(t)
\end{array}\right] \\
& \left.\left(\frac{1}{\operatorname{RePr}} \frac{\partial \theta}{\partial n}(\xi, t)\right)\right|_{\Gamma_{H}}=\left[b_{\theta_{H}}(\xi) b_{d \theta_{H}}(\xi)\right]\left[\begin{array}{l}
u_{b \theta_{H}}(t) \\
u_{d \theta_{H}}(t)
\end{array}\right] \\
& \left.(\mathcal{T}(v(\xi, t), p(\xi, t)) \cdot n)\right|_{\Gamma_{O}}=0,\left.\quad v(\xi, t)\right|_{\Gamma_{W}}=0 \\
& \left.\frac{\partial \theta}{\partial n}(\xi, t)\right|_{\Gamma_{O}}=0,\left.\quad \theta(\xi, t)\right|_{\left(\Gamma_{W} \backslash \Gamma_{H}\right)}=0
\end{aligned}
$$

where $n$ denotes the unit outward normal vector of $\Gamma, \mathcal{T}$ is the fluid Cauchy stress tensor and $\alpha_{v}, \alpha_{\theta} \geq 0$ are constants, $u_{b}=\left[u_{b v_{I}}, u_{b \theta_{I}}, u_{b \theta_{H}}\right]^{\mathrm{T}}$ are control inputs, $u_{d}=\left[u_{d v_{I}}, u_{d \theta_{I}}, u_{d \theta_{H}}\right]^{\mathrm{T}}$ are disturbance inputs, and the control and the disturbance inputs are applied via the shape functions $b_{v_{I}}, b_{\theta_{I}}, b_{\theta_{H}}, b_{d v_{I}}, b_{d \theta_{I}}$ and $b_{d \theta_{H}}$. The inputs $u_{b}(t)$ are not directly generated by the controller, but are rather given as the output of the finite-dimensional actuator

$$
\begin{aligned}
& \dot{x}_{a}(t)=A_{a} x_{a}(t)+B_{a} u(t), \quad x_{a}(0)=x_{a 0} \in \mathbb{R}^{n_{a}}, \\
& u_{b}(t)=C_{a} x_{a}(t),
\end{aligned}
$$




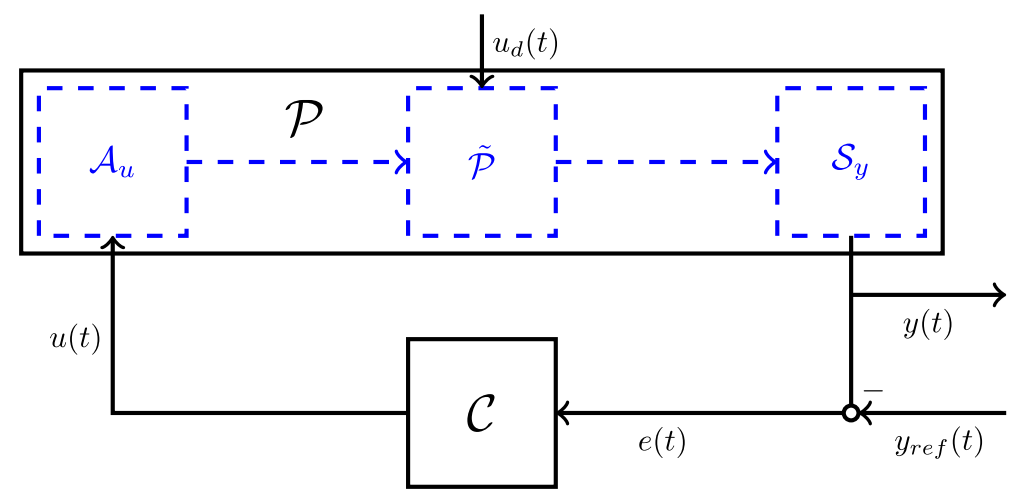

Fig. 2 A closed-loop control scheme with an actuator $\mathcal{A}_{u}$, a plant $\tilde{\mathcal{P}}$, a sensor $\mathcal{S}_{y}$, the cascade system $\mathcal{P}$ and a controller $\mathcal{C}$

which takes as its input the control signal $u(t)$ generated by the controller.

We are mainly interested in two types of observations. These are weighted temperature or velocity averages either over a two-dimensional domain $\Omega_{C} \subset \Omega$, given by

$$
y_{\Omega}(t)=\left\langle c_{\Omega}(\xi),[v(\xi, t) \theta(\xi, t)]^{\mathrm{T}}\right\rangle_{\Omega_{C}},
$$

or over a one-dimensional domain $\Gamma_{C} \subset \Gamma$, given by

$$
y_{\Gamma}(t)=\left\langle c_{\Gamma}(\xi),[v(\xi, t) \theta(\xi, t)]^{\mathrm{T}}\right\rangle_{\Gamma_{C}},
$$

and we denote by $y_{b}$ the observation of interest consisting of a combination of the two types. Note that one may include several observations of one type with the restriction that one needs to increase the number of inputs $u(t)$ accordingly to at least match the number of observations, cf. Assumption 3.1 in Sect. 3. These additional inputs are included in (5d)-(5f) by considering vector valued $u_{b v_{I}}, u_{b \theta_{I}}, u_{b_{\theta_{H}}}, b_{v_{I}}, b_{\theta_{I}}$ and $b_{\theta_{H}}$. Just as in the case of the fluid input, the fluid output is also processed by a finite-dimensional system, the sensor

$$
\begin{aligned}
\dot{x}_{s}(t) & =A_{s} x_{s}(t)+B_{s} y_{b}(t), \quad x_{s}(0)=x_{s 0} \in \mathbb{R}^{n_{s}}, \\
y(t) & =C_{s} x_{s}(t)
\end{aligned}
$$

with the observation $y(t)$. The complete plant thus consists of the linearized translated Boussinesq equations (5) coupled with the actuator (6) via the input $u_{b}$ and with the sensor (8) via the output $y_{b}$. Recall that we refer to the system (5)-(8) as the cascade system. Figure 2 depicts the control scheme consisting of the cascade system and an error feedback controller.

The control goal is considered for the observation $y(t)$ of the sensor, which we want to converge exponentially to a prescribed reference trajectory $y_{\mathrm{ref}}(t)$ of the form 
(2) despite the disturbance signal $u_{d}(t)$ given by (3). That is, for some $M_{r}, \omega_{r}>0$ it should hold that

$$
\left\|y(t)-y_{\mathrm{ref}}(t)\right\| \leq M_{r} \mathrm{e}^{-\omega_{r} t} P_{0}
$$

where $P_{0}$ is determined by the initial states of the linearized translated Boussinesq equations, the actuator, the sensor and the controller and the coefficients $a_{i}(t), b_{i}(t)$, $c_{i}(t)$ and $d_{i}(t)$ of the reference signal (2) and the disturbance signal (3).

\subsection{Abstract formulation of the control system}

The controller to be implemented achieves output tracking for a class of abstract linear systems, which motivates us to present the cascade system as one. We define the system dynamics operator based on a weak formulation of the cascade system. We then follow up with formulation of the operators related to the abstract boundary control system representation of the cascade system, a formulation choice natural in the presence of boundary inputs such as (5d)-(5f). Finally, we connect the abstract boundary control system framework to the abstract state space formulation of the cascade system.

To prepare for the formulations, we define the spaces

$$
\begin{aligned}
X_{v} & =\left\{v \in\left(L^{2}(\Omega)\right)^{2}|\nabla \cdot v=0,(v \cdot n)|_{\Gamma_{W}}=0\right\}, \\
X_{b} & =X_{v} \times L^{2}(\Omega), \\
X_{\Gamma} & =\left(L^{2}\left(\Gamma_{I}\right)\right)^{2} \times L^{2}\left(\Gamma_{I}\right) \times L^{2}\left(\Gamma_{H}\right), \\
H_{v} & =\left\{v \in\left(H^{1}(\Omega)\right)^{2}|\nabla \cdot v=0, v|_{\Gamma_{W}}=0\right\}, \\
H_{\theta} & =\left\{\theta \in H^{1}(\Omega)|\theta|_{\left(\Gamma_{W} \backslash \Gamma_{H}\right)}=0\right\}, \\
H_{b} & =H_{v} \times H_{\theta}
\end{aligned}
$$

concerning the Boussinesq equations and the spaces

$$
\begin{aligned}
& X=X_{b} \times \mathbb{R}^{n_{a}} \times \mathbb{R}^{n_{s}}, \\
& H=H_{b} \times \mathbb{R}^{n_{a}} \times \mathbb{R}^{n_{s}}
\end{aligned}
$$

concerning the cascade system. Furthermore, for all $x=\left[x_{b}, x_{a}, x_{s}\right]^{\mathrm{T}} \in X$, where $x_{b}=[v, \theta]^{\mathrm{T}}$, we define the norms

$$
\begin{aligned}
\|x\|_{X}^{2} & =\left\|x_{b}\right\|_{X_{b}}^{2}+\left\|x_{a}\right\|_{\mathbb{R}^{n_{a}}}^{2}+\left\|x_{s}\right\|_{\mathbb{R}^{n_{s}}}^{2}, \\
\|x\|_{H}^{2} & =\left\|x_{b}\right\|_{H_{b}}^{2}+\left\|x_{a}\right\|_{\mathbb{R}^{n_{a}}}^{2}+\left\|x_{s}\right\|_{\mathbb{R}^{n_{s}}}^{2},
\end{aligned}
$$

and denote the input space $U=\mathbb{R}^{m}$, the output space $Y=\mathbb{R}^{p_{Y}}$ and the disturbance space $U_{d}=\mathbb{R}^{d}$.

The presented observations (7) are not the only possible choices, and before focusing on the system as a whole we present an assumption characterizing the class of suitable observations 


$$
y_{b}(t)=C_{b} x_{b}(t)
$$

on the linearized Boussinesq equations.

Assumption 2.1 The observation operator satisfies $C_{b} \in \mathcal{L}\left(H_{b}, Y_{b}\right)$ for some $Y_{b}:=$ $\mathbb{R}^{p_{b}}$.

Lemma 2.2 For system (5), both the observation $y_{\Omega}=\left\langle c_{\Omega}, x_{b}\right\rangle_{\Omega_{C}}$ and $y_{\Gamma}=$ $\left\langle c_{\Gamma}, x_{b}\right\rangle_{\Gamma_{C}}$ in (7) with $c_{\Omega} \in\left(L^{2}\left(\Omega_{C}\right)\right)^{2} \times L^{2}\left(\Omega_{C}\right)$ and $c_{\Gamma} \in\left(L^{2}\left(\Gamma_{C}\right)\right)^{2} \times L^{2}\left(\Gamma_{C}\right)$ satisfy Assumption 2.1.

Proof Clearly $\left\langle c_{\Omega}, \cdot\right\rangle_{\Omega_{C}} \in \mathcal{L}\left(X_{b}, \mathbb{R}\right)$. Due to properties of the trace operator, we have for a constant $k>0$

$$
\left\langle c_{\Gamma}, x_{b}\right\rangle_{\Gamma_{C}} \leq\left\|c_{\Gamma}\right\|_{L^{2}\left(\Gamma_{C}\right)}\left\|x_{b}\right\|_{L^{2}\left(\Gamma_{C}\right)} \leq k\left\|x_{b}\right\|_{H^{1}(\Omega)},
$$

thus $\left\langle c_{\Gamma}, \cdot\right\rangle_{\Gamma_{C}} \in \mathcal{L}\left(H_{b}, \mathbb{R}\right)$.

Existence and uniqueness of steady-state solutions for the Boussinesq equations are outside the scope of this work. For the following analysis of the cascade system, we assume that a weak steady-state solution

$$
\left(w_{s s}, q_{s s}, T_{s s}\right) \in H_{v} \times L^{2}(\Omega) \times H_{\theta}
$$

for the Boussinesq equations (4) exists. Discussion on existence and uniqueness of steady-state solutions for the Boussinesq equations can be found in, e.g., [29].

As the first step toward abstract formulation of the cascade system, we construct the system dynamics operator $A$ via a weak formulation of the cascade system and verify that it generates a strongly continuous semigroup on $X$. To that end, we define the bilinear and trilinear forms

$$
\begin{aligned}
& a_{v}(v, \psi)=\frac{2}{\operatorname{Re}}\langle\epsilon(v), \epsilon(\psi)\rangle_{\Omega}+\alpha_{v}\langle v, \psi\rangle_{\Gamma_{I}} \quad \forall v, \psi \in H_{v}, \\
& a_{\theta}(\theta, \phi)=\frac{1}{\operatorname{Re} \operatorname{Pr}}\langle\nabla \theta, \nabla \phi\rangle_{\Omega}+\alpha_{\theta}\langle\theta, \phi\rangle_{\Gamma_{I}} \quad \forall \theta, \phi \in H_{\theta}, \\
& b_{v}\left(v_{1}, v_{2}, \psi\right)=\left\langle\left(v_{1} \cdot \nabla\right) v_{2}, \psi\right\rangle_{\Omega} \quad \forall v_{1}, v_{2}, \psi \in H_{v}, \\
& b_{\theta}(v, \theta, \phi)=\langle v \cdot \nabla \theta, \phi\rangle_{\Omega} \quad \forall v \in X_{v}, \forall \theta, \phi \in H_{\theta}, \\
& b_{0}(\theta, \psi)=\left\langle\hat{e}_{2} \frac{G r}{\operatorname{Re}^{2}} \theta, \psi\right\rangle_{\Omega} \quad \forall \theta \in L^{2}(\Omega), \forall \psi \in\left(L^{2}(\Omega)\right)^{2} .
\end{aligned}
$$

Here,

$$
\epsilon(v)=\frac{1}{2}\left(\nabla v+(\nabla v)^{\mathrm{T}}\right) \quad \forall v \in\left(H^{1}(\Omega)\right)^{2},
$$

thus the Cauchy stress tensor is given by

$$
\mathcal{T}(v, p)=\frac{2}{R e} \epsilon(v)-p I .
$$


Note that $0=-a_{\theta}(\theta, \phi)$ corresponds to a weak formulation of the stationary diffusion equation for the temperature subject to (5e), (5f) and (5h) with zero control and disturbance, i.e., when

$$
\left.\left(\frac{1}{\operatorname{RePr}} \frac{\partial \theta}{\partial n}+\alpha_{\theta} \theta\right)\right|_{\Gamma_{I}}=0,\left.\quad\left(\frac{1}{\operatorname{RePr}} \frac{\partial \theta}{\partial n}\right)\right|_{\Gamma_{H}}=0 .
$$

Similarly, due to Stokes formula and incompressibility, $0=-a_{v}(v, \psi)$ corresponds to a weak formulation of the stationary Stokes equation subject to (5d) and (5g) with zero control and disturbance, i.e., when

$$
\left.\left(\mathcal{T}(v, p) \cdot n+a_{v} v\right)\right|_{\Gamma_{I}}=0
$$

Consider the cascade system (5)-(8) subject to a constant boundary disturbance signal $u_{d}^{\prime}=\left[u_{d v_{I}}^{\prime}, u_{d \theta_{I}}^{\prime}, u_{d \theta_{H}}^{\prime}\right]^{\mathrm{T}}$ and denote $g_{d v_{I}}=b_{v_{I}} u_{b v_{I}}^{\prime}, g_{d \theta_{I}}=b_{\theta_{I}} u_{d \theta_{I}}^{\prime}, g_{d \theta_{H}}=$ $b_{\theta_{H}} u_{d \theta_{H}}^{\prime}$. Now the boundary conditions $(5 \mathrm{~d})-(5 \mathrm{f})$ for the cascade system are

$$
\begin{aligned}
& \left.\left(\mathcal{T}(v, p) \cdot n+\alpha_{v} v\right)\right|_{\Gamma_{I}}=b_{v} C_{a_{v}} x_{a}+g_{d v_{I}}, \\
& \left.\left(\frac{1}{\operatorname{RePr}} \frac{\partial \theta}{\partial n}+\alpha_{\theta} \theta\right)\right|_{\Gamma_{I}}=b_{\theta_{I}} C_{a_{\theta_{I}}} x_{a}+g_{d \theta_{I}}, \\
& \left.\left(\frac{1}{\operatorname{RePr}} \frac{\partial \theta}{\partial n}\right)\right|_{\Gamma_{H}}=b_{\theta_{H}} C_{a_{\theta_{H}}} x_{a}+g_{d \theta_{H}},
\end{aligned}
$$

where $C_{a_{v}}, C_{a_{\theta_{I}}}$ and $C_{a_{\theta_{H}}}$ are obtained from $C_{a}=\left[C_{a_{v}}, C_{a_{\theta_{I}}}, C_{a_{\theta_{H}}}\right]^{\mathrm{T}}$. A weak formulation for a steady-state solution of the cascade system subject to $u_{d}^{\prime}$ and a constant control input $u^{\prime}$ is now given by

$$
\begin{aligned}
0= & -a_{v}(v, \psi)-b_{v}\left(v, w_{s s}, \psi\right)-b_{v}\left(w_{s s}, v, \psi\right)+b_{0}(\theta, \psi)+\langle\mathcal{T}(v, p) \cdot n, \psi\rangle_{\Gamma_{I}} \\
& +\alpha_{v}\langle v, \psi\rangle_{\Gamma_{I}} \\
= & -a_{v}(v, \psi)-b_{v}\left(v, w_{s s}, \psi\right)-b_{v}\left(w_{s s}, v, \psi\right)+b_{0}(\theta, \psi) \\
& +\left\langle b_{v} C_{a_{v}} x_{a}+g_{d v_{I}}, \psi\right\rangle_{\Gamma_{I}} \forall \psi \in H_{v}, \\
0= & -a_{\theta}(\theta, \phi)-b_{\theta}\left(w_{s s}, \theta, \phi\right)-b_{\theta}\left(v, T_{s s}, \phi\right)+\frac{1}{\operatorname{RePr}}\left\langle\frac{\partial \theta}{\partial n}, \phi\right\rangle_{\Gamma_{I} \cup \Gamma_{H}}+\alpha_{\theta}\langle\theta, \phi\rangle_{\Gamma_{I}} \\
= & -a_{\theta}(\theta, \phi)-b_{\theta}\left(w_{s s}, \theta, \phi\right)-b_{\theta}\left(v, T_{s s}, \phi\right)+\left\langle b_{\theta_{I}} C_{\theta_{I}} x_{a}+g_{d \theta_{I}}, \phi\right\rangle_{\Gamma_{I}} \\
& +\left\langle b_{\theta_{H}} C_{\theta_{H}} x_{a}+g_{d \theta_{H}}, \phi\right\rangle_{\Gamma_{H}} \quad \forall \phi \in H_{\theta}, \\
0= & \left\langle A_{a} x_{a}, \psi_{a}\right\rangle_{\mathbb{R}^{n_{a}}}+\left\langle B_{a} u^{\prime}, \psi_{a}\right\rangle_{\mathbb{R}^{n_{a}}} \quad \forall \psi_{a} \in \mathbb{R}^{n_{a}}, \\
0= & \left\langle A_{s} x_{s}, \psi_{s}\right\rangle_{\mathbb{R}^{n_{s}}}+\left\langle B_{s} C_{b}\left[\begin{array}{l}
v \\
\theta
\end{array}\right], \psi_{s}\right\rangle_{\mathbb{R}^{n_{s}}} \quad \forall \psi_{s} \in \mathbb{R}^{n_{s}} .
\end{aligned}
$$

Motivated by the weak formulation, we define for $u_{d}^{\prime}=0$ and $u^{\prime}=0$ the bilinear form

$$
\begin{aligned}
a_{0}(\Phi, \Psi) & =a_{0}\left(\left(v, \theta, x_{a}, x_{s}\right),\left(\psi, \phi, \psi_{a}, \psi_{s}\right)\right) \\
& =a_{v}(v, \psi)+a_{\theta}(\theta, \phi)+b_{v}\left(v, w_{s s}, \psi\right)+b_{v}\left(w_{s s}, v, \psi\right)+b_{\theta}\left(w_{s s}, \theta, \phi\right)
\end{aligned}
$$




$$
\begin{aligned}
& +b_{\theta}\left(v, T_{s s}, \phi\right)-b_{0}(\theta, \psi)-\left\langle b_{v_{I}} C_{a_{v}} x_{a}, \psi\right\rangle_{\Gamma_{I}}-\left\langle b_{\theta_{I}} C_{a_{\theta_{I}}} x_{a}, \phi\right\rangle_{\Gamma_{I}} \\
& -\left\langle b_{\theta_{H}} C_{a_{\theta_{H}}} x_{a}, \phi\right\rangle_{\Gamma_{H}}-\left\langle B_{s} C_{b}[v \theta]^{\mathrm{T}}, \psi_{s}\right\rangle_{\mathbb{R}^{n_{s}}}-\left\langle A_{a} x_{a}, \psi_{a}\right\rangle_{\mathbb{R}^{n_{a}}} \\
& -\left\langle A_{s} x_{s}, \psi_{s}\right\rangle_{\mathbb{R}^{n_{s}}} \quad \forall \Phi, \Psi \in H
\end{aligned}
$$

and more generally for $u^{\prime}=0$ and some $u_{d}^{\prime} \in \mathbb{R}^{d}$ the bilinear form

$$
\begin{aligned}
a_{g}(\Phi, \Psi)= & a_{0}\left(\left(v, \theta, x_{a}, x_{s}\right),\left(\psi, \phi, \psi_{a}, \psi_{s}\right)\right)-\left\langle g_{d v_{I}}, \psi\right\rangle_{\Gamma_{I}}-\left\langle g_{d \theta_{I}}, \phi\right\rangle_{\Gamma_{I}} \\
& -\left\langle g_{d \theta_{H}}, \phi\right\rangle_{\Gamma_{H}} \forall \Phi, \Psi \in H .
\end{aligned}
$$

Using the bilinear form $a_{0}(\cdot, \cdot)$, we define the linear operator $A$ by

$$
\begin{aligned}
\langle A x, \Psi\rangle_{X} & =-a_{0}(x, \Psi) \\
D(A) & =\left\{x \in H \mid \forall \Psi \in H, \Psi \rightarrow a_{0}(x, \Psi) \text { is } X \text {-continuous }\right\} .
\end{aligned}
$$

Note that the geometry of $\Omega$ and presence of the mixed boundary conditions reduce regularity of the solutions of (5) so that $D(A) \not \subset\left(H^{2}(\Omega)\right)^{2} \times H^{2}(\Omega) \times \mathbb{R}^{n_{a}} \times \mathbb{R}^{n_{s}}$, cf. [12,24,30].

The following semigroup generation result is not only needed for the abstract system formulation but also the coercivity and boundedness results for the bilinear form will be utilized for the controller implementation to achieve output tracking, cf. [34]. Similar results focusing mainly on semigroup generation instead of coercivity and boundedness of the bilinear forms for both the linearized Boussinesq equations and the linearized incompressible Navier-Stokes equations without additional ODE dynamics have been presented in multiple papers, see, e.g., [12,24,32].

Theorem 2.3 Operator $A$ is the generator of an analytic semigroup on $X$ and the bilinearform $a_{0}(\cdot, \cdot)$ is $H$-bounded and $H$-coercive, i.e., $H$ is continuously and densely embedded in $X$ and there exist $c, \lambda, \gamma>0$ such that for all $\Phi, \Psi \in H$

$$
\begin{aligned}
\left|a_{0}(\Phi, \Psi)\right| & \leq c\|\Phi\|_{H}\|\Psi\|_{H}, \\
a_{0}(\Phi, \Phi) & \geq \gamma\|\Phi\|_{H}^{2}-\lambda\|\Phi\|_{X}^{2} .
\end{aligned}
$$

Proof Throughout the proof, we denote by $c$ a generic positive constant which may have a different value for each occurrence. We start by considering the terms $a_{\theta}(\cdot, \cdot)$ and $a_{v}(\cdot, \cdot)$. Now properties of the trace operator imply

$$
0 \leq \alpha_{\theta}\langle\theta, \theta\rangle_{\Gamma_{I}} \leq c\|\theta\|_{H^{1}}^{2}
$$

thus using Poincare's inequality we get for $\theta \in H_{\theta}$ and a constant $c_{\theta}>0$

$$
a_{\theta}(\theta, \theta)=\frac{1}{\operatorname{RePr}}\langle\nabla \theta, \nabla \theta\rangle_{\Omega}+\alpha_{\theta}\langle\theta, \theta\rangle_{\Gamma_{I}} \geq c_{\theta}\|\theta\|_{H^{1}}^{2},
$$


i.e., $a_{\theta}(\cdot, \cdot)$ is $H_{\theta}$-coercive. Since

$$
\begin{aligned}
\left|a_{\theta}(\theta, \phi)\right| & \leq\left|\frac{1}{\operatorname{RePr}}\langle\nabla \theta, \nabla \phi\rangle_{\Omega}\right|+\left|\alpha_{\theta}\langle\theta, \phi\rangle_{\Gamma_{I}}\right| \\
& \leq \frac{1}{\operatorname{RePr}}\|\theta\|_{H^{1}}\|\phi\|_{H^{1}}+c\|\theta\|_{H^{1}}\|\phi\|_{H^{1}},
\end{aligned}
$$

$a_{\theta}(\cdot, \cdot)$ is also $H_{\theta}$-bounded.

Regarding $a_{v}(\cdot, \cdot)$, it similarly holds that

$$
0 \leq \alpha_{v}\langle v, v\rangle_{\Gamma_{I}} \leq c\|v\|_{H^{1}}^{2}
$$

and the norm $\|\epsilon(\cdot)\|_{L^{2}}$ is equivalent to the norm $\|\cdot\|_{H^{1}}$ through Korn's and Poincare's inequalities. Now for $v \in H_{v}$ and a constant $c_{v}>0$

$$
a_{v}(v, v)=\frac{2}{R e}\langle\epsilon(v), \epsilon(v)\rangle_{\Omega}+\alpha_{v}\langle v, v\rangle_{\Gamma_{I}} \geq \frac{2}{R e}\|\epsilon(v)\|_{L^{2}}^{2} \geq c_{v}\|v\|_{H^{1}}^{2},
$$

thus $a_{v}(\cdot, \cdot)$ is $H_{v}$-coercive. Since additionally

$$
\begin{aligned}
\left|a_{v}(v, \psi)\right| & \leq\left|\frac{2}{\operatorname{Re}}\langle\epsilon(v), \epsilon(\psi)\rangle_{\Omega}\right|+\left|\alpha_{v}\langle v, \psi\rangle_{\Gamma_{I}}\right| \\
& \leq c\left(\|v\|_{H^{1}}\|\psi\|_{H^{1}}+\|v\|_{H^{1}}\|\psi\|_{H^{1}}\right),
\end{aligned}
$$

$a_{v}(\cdot, \cdot)$ is also $H_{v}$-bounded. Combining (17)-(20), we have that there exist constants $c_{1}, \gamma_{1}>0$ such that for all $\phi_{b}, \psi_{b} \in H_{b}$ the bilinear form

$$
a_{1}\left(\psi_{b}, \phi_{b}\right)=a_{1}((v, \theta),(\psi, \phi)):=a_{v}(v, \psi)+a_{\theta}(\theta, \phi)
$$

satisfies

$$
\begin{aligned}
\left|a_{1}\left(\phi_{b}, \psi_{b}\right)\right| & \leq c_{1}\left\|\phi_{b}\right\|_{H_{b}}\left\|\psi_{b}\right\|_{H_{b}}, \\
a_{1}\left(\phi_{b}, \phi_{b}\right) & \geq \gamma_{1}\left\|\phi_{b}\right\|_{H_{b}}^{2} .
\end{aligned}
$$

The rest of the proof now consists of presenting estimates for the norms of the remaining terms of $a_{0}(\cdot, \cdot)$.

We immediately have that

$$
\begin{aligned}
& \left|b_{0}(\theta, \psi)\right| \leq c\|\theta\|_{L^{2}}\|\psi\|_{X_{v}}, \\
& \left|\left\langle A_{a} x_{a}, \psi_{a}\right\rangle_{\mathbb{R}^{n_{a}}}\right| \leq c\left\|x_{a}\right\|_{\mathbb{R}^{n_{a}}}\left\|\psi_{a}\right\|_{\mathbb{R}^{n_{a}}}, \\
& \left|\left\langle A_{s} x_{s}, \psi_{s}\right\rangle_{\mathbb{R}^{n_{s}}}\right| \leq c\left\|x_{s}\right\|_{\mathbb{R}^{n_{s}}}\left\|\psi_{s}\right\|_{\mathbb{R}^{n_{s}}} .
\end{aligned}
$$

Regarding the form $b_{\theta}(\cdot, \cdot, \cdot)$, by Sobolev embeddings, $L^{2}$-duality of $H^{1 / 2}$ and $H^{-1 / 2}$ and Ladyzhenskaya's inequality 


$$
\begin{aligned}
& \left|b_{\theta}\left(v, T_{s s}, \theta\right)\right| \\
& =\left|\left\langle v \cdot \nabla T_{s s}, \theta\right\rangle_{\Omega}\right| \\
& \leq\left|\left\langle v T_{s s}, \nabla \theta\right\rangle_{\Omega}\right|+\left|\left\langle v \cdot n, T_{s s} \theta\right\rangle_{\Gamma}\right| \\
& \leq\left\|v T_{S S}\right\|_{L^{2}}\|\nabla \theta\|_{L^{2}}+c\|v\|_{H^{1}}\left\|T_{S S} \theta\right\|_{L^{2}} \\
& \leq\|v\|_{L^{4}}\left\|T_{s S}\right\|_{L^{4}}\|\nabla \theta\|_{L^{2}}+c\|v\|_{H^{1}}\left\|T_{S S}\right\|_{L^{4}}\|\theta\|_{L^{4}} \\
& \leq c\left\|T_{S s}\right\|_{H^{1}}\left(\|v\|_{L^{2}}^{1 / 2}\|\nabla v\|_{L^{2}}^{1 / 2}\|\nabla \theta\|_{L^{2}}+\|v\|_{H^{1}}\|\theta\|_{L^{2}}^{1 / 2}\|\nabla \theta\|_{L^{2}}^{1 / 2}\right) .
\end{aligned}
$$

Similarly, we have

$$
\begin{aligned}
\left|b_{\theta}\left(w_{s s}, \theta, \theta\right)\right| & =\frac{1}{2}\left\langle w_{s s}, \nabla \theta^{2}\right\rangle_{\Omega} \\
& =\frac{1}{2}\left\langle w_{s s} \cdot n, \theta^{2}\right\rangle_{\Gamma} \\
& \leq c\left\|w_{s s}\right\|_{H^{1}}\left\|\theta^{2}\right\|_{L^{2}} \\
& =c\left\|w_{s s}\right\|_{H^{1}}\|\theta\|_{L^{4}}^{2} \\
& \leq c\|\theta\|_{L^{2}}\|\nabla \theta\|_{L^{2}}
\end{aligned}
$$

Furthermore,

$$
\begin{aligned}
\left|b_{\theta}(v, \theta, \phi)\right| & \leq\left|\langle v, \nabla(\theta \phi)\rangle_{\Omega}\right|+\left|\langle v \theta, \nabla \phi\rangle_{\Omega}\right| \\
& =\left|\langle v \cdot n, \theta \phi\rangle_{\Gamma}\right|+\left|\langle v \theta, \nabla \phi\rangle_{\Omega}\right| \\
& \leq c\|v\|_{H^{1}}\|\theta\|_{H^{1}}\|\phi\|_{H^{1}} .
\end{aligned}
$$

Regarding the form $b_{v}(\cdot, \cdot, \cdot)$, we again use $L^{2}$-duality of $H^{1 / 2}$ and $H^{-1 / 2}$, Sobolev embeddings and Ladyzhenskaya's inequality to form the estimates. Now

$$
\begin{aligned}
\left|b_{v}\left(w_{s s}, v, v\right)\right| & =\left|\left\langle\left(w_{s s} \cdot \nabla\right) v, v\right\rangle_{\Omega}\right| \\
& \leq\left|\left\langle w_{s s},(v \cdot \nabla) v\right\rangle_{\Omega}\right|+\left|\left\langle w_{s s} \cdot n, v \cdot v\right\rangle_{\Gamma}\right| \\
& \leq c\left(\left\|w_{s s}\right\|_{L^{4}}\|v\|_{L^{4}}\|\nabla v\|_{L^{2}}+\left\|w_{s s}\right\|_{H^{1}}\|v\|_{L^{4}}^{2}\right) \\
& \leq c\left(\|v\|_{L^{2}}^{1 / 2}\|\nabla v\|_{L^{2}}^{3 / 2}+\|v\|_{L^{2}}\|\nabla v\|_{L^{2}}\right)
\end{aligned}
$$

and

Additionally,

$$
\begin{aligned}
\left|b_{v}\left(v, w_{s s}, v\right)\right| & =\left|\left\langle(v \cdot \nabla) w_{s s}, v\right\rangle_{\Omega}\right| \\
& \leq\|v\|_{L^{4}}^{2}\left\|\nabla w_{s s}\right\|_{L^{2}} \\
& \leq c\left\|w_{s s}\right\|_{H^{1}}\|v\|_{L^{2}}\|\nabla v\|_{L^{2}}
\end{aligned}
$$

$$
\begin{aligned}
\left|b_{v}\left(v_{1}, v_{2}, \psi\right)\right| & \leq\left|\left\langle v_{1},\left(v_{2} \cdot \nabla\right) \psi\right\rangle_{\Omega}\right|+\left|\left\langle v_{1} \cdot n, v_{2} \cdot \psi\right\rangle_{\Gamma}\right| \\
& \leq c\left\|v_{1}\right\|_{L^{4}}\left\|v_{2}\right\|_{L^{4}}\|\nabla \psi\|_{L^{2}}+c\left\|v_{1}\right\|_{H^{1}}\left\|v_{2} \cdot \psi\right\|_{L^{2}} \\
& \leq c\left(\left\|v_{1}\right\|_{H^{1}}\left\|v_{2}\right\|_{H^{1}}\|\psi\|_{H^{1}}+\left\|v_{1}\right\|_{H^{1}}\left\|v_{2}\right\|_{L^{4}}\|\psi\|_{L^{4}}\right) \\
& \leq c\left\|v_{1}\right\|_{H^{1}}\left\|v_{2}\right\|_{H^{1}}\|\psi\|_{H^{1}} .
\end{aligned}
$$


Finally, properties of the trace operator together with Assumption 2.1 and duality imply

$$
\begin{aligned}
& \left|\left\langle b_{t} C_{a} \psi_{a}, \psi_{b}\right\rangle_{\Gamma}\right| \leq c\left\|\psi_{a}\right\|_{\mathbb{R}^{n_{a}}}\left\|\psi_{b}\right\|_{H_{b}}, \\
& \left|\left\langle B_{s} C_{b} \psi_{b}, \psi_{s}\right\rangle_{\mathbb{R}^{n_{s}}}\right| \leq c\left\|\phi_{s}\right\|_{\mathbb{R}^{n_{s}}}\left\|\psi_{b}\right\|_{H_{b}},
\end{aligned}
$$

where $b_{t}:=\left[b_{v_{I}}, b_{\theta_{I}}, b_{\theta_{H}}\right]$. Recalling the norm definitions (11), equations (21a), (22)-(24), (27) and (30)-(32) together imply $H$-boundedness of $a_{0}(\cdot, \cdot) . H$-coercivity of $a_{0}(\cdot, \cdot)$ follows from (21b) after applying Young's inequality to (25), (26), (28), (29), (31) and (32). Finally, $H$-coercivity and $H$-boundedness of $a_{0}(\cdot, \cdot)$ imply generation of an analytic semigroup on $X$, see, e.g., [6].

To formulate the cascade system as an abstract boundary control system in the sense of [16, Ch. 3.3], we next define the related operators. In what follows, $\mathbb{P}$ denotes the Leray projector as defined in [32, Lemma 2.2], which is used to eliminate the pressure term while imposing incompressibility. Define the operators

$$
\begin{aligned}
& \mathcal{A}\left[\begin{array}{c}
v \\
\theta \\
x_{a} \\
x_{s}
\end{array}\right]=\left[\begin{array}{c}
\mathbb{P}\left(\frac{1}{R e} \Delta v-\left(w_{s s} \cdot \nabla\right) v-(v \cdot \nabla) w_{s s}+\frac{G r}{R e^{2}} \hat{e}_{2} \theta\right) \\
\frac{1}{\operatorname{RePr}} \Delta \theta-w_{s s} \cdot \nabla \theta-v \cdot \nabla T_{s s} \\
A_{a} x_{a} \\
A_{s} x_{s}+B_{s} C_{b}[v, \theta]^{\mathrm{T}}
\end{array}\right]: D(\mathcal{A}) \rightarrow X, \\
& B_{\Gamma_{u}}=\left[\begin{array}{ccc}
b_{v_{I}} & 0 & 0 \\
0 & b_{\theta_{I}} & 0 \\
0 & 0 & b_{\theta_{H}}
\end{array}\right]: U_{b} \rightarrow X_{\Gamma}, \\
& B_{\Gamma_{u_{d}}}=\left[\begin{array}{ccc}
b_{d v_{I}} & 0 & 0 \\
0 & b_{d \theta_{I}} & 0 \\
0 & 0 & b_{d \theta_{H}}
\end{array}\right]: U_{d} \rightarrow X_{\Gamma}, \\
& B_{\Gamma}=\left[\begin{array}{ll}
B_{\Gamma_{u}} & B_{\Gamma_{u_{d}}}
\end{array}\right] \text {, }
\end{aligned}
$$

where $U_{b}:=\mathbb{R}^{m_{b}}$. Operator $\mathcal{A}$ coincides with $A$ on $D(A)$ but has a larger domain due to relaxed boundary conditions within the boundary parts $\Gamma_{I}$ and $\Gamma_{H}$ affected by disturbance inputs. That is, noting that the Neumann trace of $H_{b}$ functions is in $\left(H^{-1 / 2}(\Gamma)\right)^{2} \times H^{-1 / 2}(\Gamma)$ and recalling the definition of $a_{g}(\cdot, \cdot)$ in (15), the domain is given by

$$
\begin{aligned}
D(\mathcal{A})= & \left\{x \in H \mid \exists g_{d v_{I}} \in\left(H^{-1 / 2}\left(\Gamma_{I}\right)\right)^{2}, \exists g_{d \theta_{I}} \in H^{-1 / 2}\left(\Gamma_{I}\right),\right. \\
& \left.\exists g_{d \theta_{H}} \in H^{-1 / 2}\left(\Gamma_{H}\right): \forall \Psi \in H, \Psi \rightarrow a_{g}(x, \Psi) \text { is } X \text {-continuous }\right\} .
\end{aligned}
$$

Corresponding to the control and disturbance boundary conditions (5d)-(5f), we define the operator

$$
\tilde{\mathcal{B}}_{b}\left[\begin{array}{c}
v \\
\theta \\
p
\end{array}\right]=\left[\begin{array}{c}
\left.\left(\mathcal{T}(v, p) \cdot n+\alpha_{v} v\right)\right|_{\Gamma_{I}} \\
\left.\left(\frac{1}{\operatorname{RePr}} \frac{\partial \theta}{\partial n}+\alpha_{\theta} \theta\right)\right|_{\Gamma_{I}} \\
\left.\left(\frac{1}{\operatorname{RePr}} \frac{\partial \theta}{\partial n}\right)\right|_{\Gamma_{H}}
\end{array}\right]: D\left(\tilde{\mathcal{B}}_{b}\right) \subset X_{b} \times L^{2}(\Omega) \rightarrow X_{\Gamma}
$$


The pressure $p$ is uniquely defined by the velocity $v$, see [4], thus there exists an operator $\mathcal{B}_{b}$ such that

$$
\mathcal{B}_{b}\left[\begin{array}{l}
v \\
\theta
\end{array}\right]=\tilde{\mathcal{B}}_{b}\left[\begin{array}{l}
v \\
\theta \\
p
\end{array}\right] \quad \forall\left[\begin{array}{l}
v \\
\theta \\
p
\end{array}\right] \in D\left(\tilde{\mathcal{B}}_{b}\right), \quad D\left(\mathcal{B}_{b}\right)=\left\{(v, \theta) \in D\left(\tilde{\mathcal{B}}_{b}\right)\right\}
$$

To match the state variable $x \in X$ of the cascade system, we finally define the operator

$$
\mathcal{B}=\left[\begin{array}{lll}
\mathcal{B}_{b} & 0 & 0
\end{array}\right]: D(\mathcal{B})=D\left(\mathcal{B}_{b}\right) \times \mathbb{R}^{n_{a}} \times \mathbb{R}^{n_{s}} \subset X \rightarrow X_{\Gamma}
$$

Since $A=\left.\mathcal{A}\right|_{\mathcal{N}\left(\mathcal{B}-\left[0, B_{\Gamma_{u}} C_{a}, 0\right]\right)}$ generates an analytic semigroup on $X$ by Theorem 2.3 and $\mathcal{B}$ is onto $X_{\Gamma}$, cf. [12], by defining

$$
B=\left[\begin{array}{lll}
0_{X_{b}} & B_{a} & 0_{\mathbb{R}^{n_{s}}}
\end{array}\right]^{\mathrm{T}} \in \mathcal{L}(U, X)
$$

we have that the cascade system (5)-(8) corresponds to the abstract boundary control system

$$
\begin{aligned}
\dot{x}(t) & =\mathcal{A} x(t)+B u(t), \\
\mathcal{B} x(t) & =B_{\Gamma}\left[\begin{array}{c}
C_{a} x_{a}(t) \\
u_{d}(t)
\end{array}\right]
\end{aligned}
$$

on $X$ with the (boundary) input space $X_{\Gamma}$ in the sense of [16, Ch. 3.3]. The system observation is given by

$$
y(t)=C x(t), \quad C=\left[0_{X_{b}}, 0_{\mathbb{R}^{n a}}, C_{s}\right] \in \mathcal{L}(X, Y) .
$$

Note that the control and observation operators of the boundary control system are bounded and the disturbance $B_{\Gamma_{u_{d}}} u_{d}$ is the only boundary input of the boundary control system. The boundary control system formulation of the cascade system also has the following equivalent state space formulation.

Proposition 2.4 The cascade system (5)-(8) can be formulated as

$$
\begin{aligned}
& \dot{x}(t)=A x(t)+B u(t)+B_{d} u_{d}(t), \quad x(0)=x_{0} \in X, \\
& y(t)=C x(t)+D_{d} u_{d}(t),
\end{aligned}
$$

where the dynamics operator A defined as in (16) generates an analytic semigroup on the state space $X$ defined in (10a) and the control operator $B$ together with the observation operator $C$ defined in (33) and (34c) are bounded. Additionally, a change of the state variable $x$ can be applied such that also the resulting disturbance operator $B_{d}$ is bounded. 
Proof Existence of the state space formulation (35) follows from the boundary control system formulation as presented in [40, Ch. 10] and boundedness of $B$ and $C$ is apparent from their definitions. The change of variable $\tilde{x}_{b}=x_{b}-B_{I} B_{\Gamma_{u_{d}}}$, where $B_{I} \in \mathcal{L}\left(X_{\Gamma}, D\left(\mathcal{A}_{b}\right)\right)$ is a right inverse of $\mathcal{B}_{b}$ used to homogenize the boundary conditions and obtain a bounded operator $B_{d}$, is presented in [16, Ch. 3.3]. Note that the change of variable utilizes smoothness of the disturbance signal $u_{d}$ given by (3) and introduces a bounded feedthrough operator $D_{d}$ into the system.

The state space formulation together with the fact that the operators $B, B_{d}, C$ and $D_{d}$ are bounded will later in this paper be utilized for implementing the output tracking controller.

Remark 2.5 The controller to be implemented uses no information on the disturbance related operators $B_{d}$ and $D_{d}$, thus we do not formulate the cascade system using the state variable $\tilde{x}_{b}$. However, one needs to verify that a representation using bounded disturbance operators $B_{d}$ and $D_{d}$ exists.

\subsection{Stabilizability and detectability of the system}

We will be using a controller including an observer-like structure, which means that we need to address both stabilizability and detectability properties of the cascade system (5)-(8). Here, we focus on deriving sufficient conditions for exponential stabilizability and exponential detectability of the cascade system based on properties of the linearized translated Boussinesq equations, the actuator and the sensor.

To begin with we note that, in addition to the cascade system, also the linearized translated Boussinesq equations (5) form an abstract boundary control system

$$
\begin{aligned}
\dot{x}_{b}(t) & =\mathcal{A}_{b} x_{b}(t), \\
\mathcal{B}_{b} x_{b}(t) & =B_{\Gamma}\left[\begin{array}{l}
u_{b}(t) \\
u_{d}(t)
\end{array}\right],
\end{aligned}
$$

which can be verified by repeating the steps of Sect. 2.2 without the actuator and sensor dynamics. Here

$$
\begin{aligned}
\mathcal{A}_{b} & =\left.\mathcal{A}\right|_{D\left(\mathcal{A}_{b}\right)}, \\
D\left(\mathcal{A}_{b}\right) & =\left\{x_{b} \in H_{b} \mid \exists g_{v_{I}} \in\left(H^{-1 / 2}\left(\Gamma_{I}\right)\right)^{2}, \exists g_{\theta_{I}} \in H^{-1 / 2}\left(\Gamma_{I}\right),\right. \\
& \left.\exists g_{\theta_{H}} \in H^{-1 / 2}\left(\Gamma_{H}\right): \forall \varphi \in H_{b}, \varphi \rightarrow a_{b}\left(x_{b}, \varphi\right) \text { is } X_{b} \text {-continuous }\right\}
\end{aligned}
$$

with the bilinear form $a_{b}(\cdot, \cdot)$ defined by

$$
\begin{aligned}
& a_{b}((v, \theta),(\psi, \phi)) \\
& =a_{v}(v, \psi)+a_{\theta}(\theta, \phi)+b_{v}\left(v, w_{s s}, \psi\right)+b_{v}\left(w_{s s}, v, \psi\right)+b_{\theta}\left(w_{s s}, \theta, \phi\right) \\
& \quad+b_{\theta}\left(v, T_{s s}, \phi\right)-b_{0}(\theta, \psi)-\left\langle g_{v_{I}}, \psi\right\rangle_{\Gamma_{I}}-\left\langle g_{\theta_{I}}, \phi\right\rangle_{\Gamma_{I}}-\left\langle g_{\theta_{H}}, \phi\right\rangle_{\Gamma_{H}} .
\end{aligned}
$$


The associated generator of a strongly continuous semigroup is $A_{b}=\left.\mathcal{A}_{b}\right|_{\mathcal{N}\left(\mathcal{B}_{b}\right)}$. Now recalling that $A=\left.\mathcal{A}\right|_{\mathcal{N}\left(\mathcal{B}-\left[0, B_{\Gamma_{u}} C_{a}, 0\right]\right)}, A$ is described by

$$
\begin{aligned}
A\left[\begin{array}{c}
x_{b} \\
x_{a} \\
x_{s}
\end{array}\right] & =\left[\begin{array}{ccc}
\mathcal{A}_{b} & 0 & 0 \\
0 & A_{a} & 0 \\
-B_{s} C_{b} & 0 & A_{s}
\end{array}\right]\left[\begin{array}{c}
x_{b} \\
x_{a} \\
x_{s}
\end{array}\right], \\
D(A) & =\left\{\left(x_{b}, x_{a}, x_{s}\right) \in D(\mathcal{A}) \mid \mathcal{B}_{b} x_{b}=B_{\Gamma_{u}} C_{a} x_{a}\right\}
\end{aligned}
$$

which is the expression that we will use for the stabilizability and detectability analysis.

Recall that by Theorem 2.3 A generates an analytic semigroup on $X$. Additionally, by Theorem 2.3, Lax-Milgram theorem and compactness of the embedding $H$ onto $X$, the resolvent of $A$ is compact on $X$, cf. [37]. Thus, $A$ has a finite number of isolated eigenvalues on the closed right half plane $\overline{\mathbb{C}_{0}^{+}}$, each with finite multiplicity. As such, stabilizability and detectability considerations of the cascade system with the bounded control operator $B$ and the bounded observation operator $C$ can be treated as controllability and observability problems of the finite-dimensional unstable part, see [16, Ch. 5.2]. That is, the pair $(C, A)$ is exponentially detectable if and only if

$$
\mathcal{N}(s I-A) \cap \mathcal{N}(C)=\{0\} \text { for all } s \in \overline{\mathbb{C}_{0}^{+}},
$$

and the pair $(A, B)$ is exponentially stabilizable if and only if

$$
\mathcal{R}(s I-A)+\mathcal{R}(B)=X \text { for all } s \in \overline{\mathbb{C}_{0}^{+}} .
$$

Recalling Assumption 2.1, the observation (12) satisfies $C_{b} \in \mathcal{L}\left(D\left(\mathcal{A}_{b}\right), Y_{b}\right)$, thus it can be included into the abstract boundary control system framework as defined in [15]. For any $s \in \rho\left(A_{b}\right)$, the transfer function $P_{b}(s)$ of the triple $\left(\mathcal{A}_{b}, \mathcal{B}_{b}, C_{b}\right)$ is then defined by

$$
P_{b}(s) u_{b}=C_{b} z(s)
$$

where $z(s) \in D\left(\mathcal{A}_{b}\right)$ is the unique solution of the abstract elliptic problem

$$
\begin{aligned}
& \mathcal{A}_{b} z(s)=s z(s), \\
& \mathcal{B}_{b} z(s)=B_{\Gamma_{u}} u_{b},
\end{aligned}
$$

see [15]. The transfer functions for the actuator (6) and the sensor (8) are defined as $P_{a}(s)=C_{a}\left(s I-A_{a}\right)^{-1} B_{a}$ and $P_{s}(s)=C_{s}\left(s I-A_{s}\right)^{-1} B_{s}$, respectively.

For ease of notation, we define the operator $B_{b}$ as

$$
B_{b}=\left(\mathcal{A}_{b}-A_{b}\right) B_{I} B_{\Gamma_{u}} \in \mathcal{L}\left(U_{b}, X_{b_{-1}}\right)
$$

where we recall that $B_{I} \in \mathcal{L}\left(X_{\Gamma}, D\left(\mathcal{A}_{b}\right)\right)$ is a right inverse of $\mathcal{B}_{b}$. Here, $A_{b}$ is regarded as an operator from $X_{b}$ to $X_{b_{-1}}$, which is the completion of $X_{b}$ with respect to the norm $\|\cdot\|_{X_{b_{-1}}}=\left\|\left(s I-A_{b}\right)^{-1}(\cdot)\right\|_{X_{b}}$ with $s \in \rho\left(A_{b}\right)$. 
Lemma 2.6 The boundary control system $\left(\mathcal{A}_{b}, \mathcal{B}_{b}, C_{b}\right)$ corresponds to a state space formulation $\left(A_{b}, B_{b}, C_{b}\right)$, which is a well-posed triple in the sense of [41, Def. 4.8]. Furthermore, the triple $\left(A_{b}, B_{b}, C_{b}\right)$ is regular in the sense of [43, Def. 4.1].

Proof The proof is a combination of existing results. We start by presenting results of [12, Sec. 2.2] relevant to the proof. Let $\lambda_{0} \in \rho\left(A_{b}\right)$ have a large enough real part. Then, the fractional powers of $\lambda_{0} I-A_{b}$ are well defined, $D\left(\left(\lambda_{0} I-A_{b}\right)^{1 / 2}\right)=H_{b}$ and

$$
\left(\lambda_{0} I-A_{b}\right)^{-1 / 4-\epsilon} B_{b} \in \mathcal{L}\left(U_{b}, X_{b}\right)
$$

for any $\epsilon>0$. Additionally, the operator $A_{b}$ has a decomposition $A_{b}=A_{b 2}+A_{b 10}$, where $A_{b 2}$ with the domain $D\left(A_{b 2}\right)=D\left(A_{b}\right)$ is self-adjoint and the generator of an exponentially stable analytic semigroup on $X_{b}$, and $A_{b 10} \in \mathcal{L}\left(H_{b}, X_{b}\right)$.

Now by [41, Prop. 6.5] both $\left(A_{b 2}, B_{b}, C_{b}\right)$ and $\left(A_{b 2}, B_{b}, A_{b 10}\right)$ are well-posed triples and even regular with zero feedthrough. We then use the feedback results of [21], which originate from [44], by considering the operator $A_{b 10}$ in the expression $A_{b}=A_{b 2}+A_{b 10}$ as an output feedback for $A_{b 2}$. By [21, Lemma 12] the triple $\left(A_{b}, B_{b}, C_{b}\right)$ is well-posed. Finally, the triple $\left(A_{b}, B_{b}, C_{b}\right)$ is regular by [41, Prop. 5.13], since the degree of unboundedness is at most $1 / 2$ for $C_{b}$ and strictly less than $1 / 2$ for $B_{b}$, cf. Assumption 2.1 and (43).

Since the transfer function $P_{b}$ is well defined, we can make the following assumptions.

Assumption 2.7 Assume that the following hold:

(i) The spectra $\sigma\left(A_{b}\right), \sigma\left(A_{a}\right)$ and $\sigma\left(A_{s}\right)$ are pairwise disjoint on $\overline{\mathbb{C}_{0}^{+}}$.

(ii) The pair $\left(C_{s}, A_{s}\right)$ is detectable.

(iii) For every $\lambda \in \sigma\left(A_{b}\right) \cap \overline{\mathbb{C}_{0}^{+}}, \mathcal{N}\left(P_{s}(\lambda) C_{b}\right) \cap \mathcal{N}\left(\lambda I-A_{b}\right)=\{0\}$.

(iv) For every $\lambda \in \sigma\left(A_{a}\right) \cap \overline{\mathbb{C}_{0}^{+}}, \mathcal{N}\left(P_{s}(\lambda) P_{b}(\lambda) C_{a}\right) \cap \mathcal{N}\left(\lambda I-A_{a}\right)=\{0\}$.

In particular, the assumption requires that also the pairs $\left(C_{b}, A_{b}\right)$ and $\left(C_{a}, A_{a}\right)$ are exponentially detectable even if $C_{b}$ is unbounded [5].

Assumption 2.8 Assume that the following hold:

(i) The spectra $\sigma\left(A_{b}\right), \sigma\left(A_{a}\right)$ and $\sigma\left(A_{s}\right)$ are pairwise disjoint on $\overline{\mathbb{C}_{0}^{+}}$.

(ii) The pair $\left(A_{a}, B_{a}\right)$ is stabilizable and $P_{a}(\lambda)$ is surjective for every $\lambda \in \sigma\left(A_{b}\right) \cap$ $\overline{\mathbb{C}_{0}^{+}}$.

(iii) For every $\lambda \in \sigma\left(A_{s}\right) \cap \overline{\mathbb{C}_{0}^{+}}, \mathcal{R}\left(\lambda I-A_{s}\right)+\mathcal{R}\left(B_{s} P_{b}(\lambda) P_{a}(\lambda)\right)=X_{s}$.

(iv) The pair $\left(\mathcal{A}_{b}, \mathcal{B}_{b}\right)$ is exponentially stabilizable, i.e., there exists $K_{b} \in$ $\mathcal{L}\left(X_{b}, U_{b}\right)$ such that $\left.\mathcal{A}_{b}\right|_{\mathcal{N}\left(\mathcal{B}_{b}-K_{b}\right)}$ generates an exponentially stable strongly continuous semigroup on $X_{b}$.

Note that due to the results in [5] we can limit our attention to considering bounded operators $K_{b}$ in Assumption 2.8(iv).

Lemma 2.9 If Assumption 2.7 holds, then the pair $(C, A)$ is exponentially detectable. 
Proof To show that (38) holds for the pair $(C, A)$, let $\left(x_{b}, x_{a}, x_{s}\right) \in \mathcal{N}(\lambda I-A) \cap \mathcal{N}(C)$, where $\lambda \in \overline{\mathbb{C}_{0}^{+}}$. Since $\left(x_{b}, x_{a}, x_{s}\right) \in D(A)$, using (34c) and (37) we have

$$
\left\{\begin{array}{l}
C_{s} x_{s}=0 \\
\left(\lambda I-A_{a}\right) x_{a}=0, \\
\left(\lambda I-\mathcal{A}_{b}\right) x_{b}=0, \\
\mathcal{B}_{b} x_{b}=B_{\Gamma_{u}} C_{a} x_{a}, \\
\left(\lambda I-A_{s}\right) x_{s}-B_{s} C_{b} x_{b}=0 .
\end{array}\right.
$$

If $\lambda \in \rho\left(A_{a}\right),(44 \mathrm{~b})$ implies $x_{a}=0$, thus (44d) implies $x_{b} \in D\left(A_{b}\right)$. If $\lambda \in \rho\left(A_{a}\right) \cap$ $\rho\left(A_{b}\right)$, then $x_{b}=0$ by (44c) and furthermore (44a), (44e) and Assumption 2.7(ii) imply $x_{s}=0$. If $\lambda \in \rho\left(A_{a}\right) \cap \sigma\left(A_{b}\right)$, then $x_{s}=\left(\lambda I-A_{s}\right)^{-1} B_{s} C_{b} x_{b}$ by $(44 \mathrm{e})$ and Assumption 2.7(i). By (44a), (44b) and Assumption 2.7(iii) we have $x_{b}=0$, which then implies $x_{s}=0$.

If $\lambda \in \sigma\left(A_{a}\right)$, then $\lambda \in \rho\left(A_{b}\right)$ by Assumption 2.7(i). Now combining (41)-(40) with (44c), (44d) and (44e) and using Assumption 2.7(i) again yields $x_{s}=(\lambda I-$ $\left.A_{s}\right)^{-1} B_{s} P_{b}(\lambda) C_{a} x_{a}$. Then, $x_{a}=0$ by (44a), (44b) and Assumption 2.7(iv). By (44d) it now holds that $x_{b} \in D\left(A_{b}\right)$, thus $x_{b}=0$ by (44c). Finally, $x_{s}=0$ by (44e). Since $\lambda \in \overline{\mathbb{C}_{0}^{+}}$was arbitrary, (38) holds and $(C, A)$ is exponentially detectable.

Lemma 2.10 If Assumption 2.8 holds, then the pair $(A, B)$ is exponentially stabilizable.

Proof We show that (39) holds for the pair $(A, B)$. Using (33) and (37), for arbitrary $z=\left(z_{b}, z_{a}, z_{s}\right) \in X$ and for any $\lambda \in \overline{\mathbb{C}_{0}^{+}}$we need to find $x=\left(x_{b}, x_{a}, x_{s}\right) \in D(A)$ and $u \in U$ such that

$$
\left\{\begin{array}{l}
\left(\lambda I-\mathcal{A}_{b}\right) x_{b}=z_{b}, \\
\left(\lambda I-A_{a}\right) x_{a}+B_{a} u=z_{a}, \\
\left(\lambda I-A_{s}\right) x_{s}-B_{s} C_{b} x_{b}=z_{s} \\
\mathcal{B}_{b} x_{b}=B_{\Gamma_{u}} C_{a} x_{a} .
\end{array}\right.
$$

If $\lambda \in \rho\left(A_{b}\right)$, there exists $\tilde{x}_{b} \in D\left(A_{b}\right)$ such that $z_{b}=\left(\lambda I-A_{b}\right) \tilde{x}_{b}=\left(\lambda I-\mathcal{A}_{b}\right) \tilde{x}_{b}$. Since $\mathcal{B}_{b} \tilde{x}_{b}=0,(45 \mathrm{a})$ and (45d) form an abstract elliptic problem

$$
\begin{aligned}
\left(\lambda I-\mathcal{A}_{b}\right)\left(x_{b}-\tilde{x}_{b}\right) & =0, \\
\mathcal{B}_{b}\left(x_{b}-\tilde{x}_{b}\right) & =B_{\Gamma_{u}} C_{a} x_{a},
\end{aligned}
$$

which has a unique solution $x_{b}-\tilde{x}_{b}=x_{b}-\left(\lambda I-A_{b}\right)^{-1} z_{b} \in D\left(\mathcal{A}_{b}\right)$ depending on $x_{a}$ [40, Rem. 10.1.5]. If in addition $\lambda \in \rho\left(A_{a}\right) \cap \rho\left(A_{s}\right)$, (45b) and (45c) yield

$$
\begin{aligned}
& x_{a}=\left(\lambda I-A_{a}\right)^{-1}\left(z_{a}-B_{a} u\right), \\
& x_{s}=\left(\lambda I-A_{s}\right)^{-1}\left(z_{s}+B_{s} C_{b} x_{b}\right),
\end{aligned}
$$


respectively, thus $x$ and $u$ for the arbitrary $z$ can be solved from (46)-(48). If $\lambda \in$ $\rho\left(A_{b}\right) \cap \rho\left(A_{s}\right) \cap \sigma\left(A_{a}\right)$, Assumption 2.8(ii) implies that there exist $x_{a}$ and $u$ such that (45b) holds. Then, $x_{b}$ and $x_{s}$ can be solved from (46) and (48). If $\lambda \in \rho\left(A_{b}\right) \cap$ $\rho\left(A_{a}\right) \cap \sigma\left(A_{s}\right)$, we can use (47), (46) and (40) to rewrite (45c) as

$$
\begin{aligned}
z_{s}= & \left(\lambda I-A_{s}\right) x_{s}-B_{s} P_{b}(\lambda) C_{a} x_{a}+B_{s} C_{b}\left(\lambda I-A_{b}\right)^{-1} z_{b} \\
= & \left(\lambda I-A_{s}\right) x_{s}+B_{s} P_{b}(\lambda) P_{a}(\lambda) u-B_{s} P_{b}(\lambda) C_{a}\left(\lambda I-A_{a}\right)^{-1} z_{a} \\
& +B_{s} C_{b}\left(\lambda I-A_{b}\right)^{-1} z_{b} .
\end{aligned}
$$

Now Assumption 2.8(iii), (46), (47) and (49) guarantee that there exist $x$ and $u$ such that (45a) holds.

By Assumption 2.8(i), the single case left to consider is $\lambda \in \sigma\left(A_{b}\right) \cap \rho\left(A_{a}\right) \cap \rho\left(A_{s}\right)$. In that case $x_{a}$ and $x_{s}$ can be solved from (47), (48) with the latter depending on the choice of $x_{b}$. By [40, Ch. 10] we have $\mathcal{A}_{b}=A_{b}+B_{b} \mathcal{B}_{b}$ on $D(\mathcal{A})$, where $A_{b}$ again denotes the extension from $X_{b}$ to $X_{b_{-1}}$ and $B_{b}$ is defined in (42). Now using (45a) and (45d) yields

$$
z_{b}=\left(\lambda I-\mathcal{A}_{b}\right) x_{b}=\left(\lambda I-A_{b}\right) x_{b}-B_{b} C_{a} x_{a}
$$

thus by (47) we have

$$
z_{b}=\left(\lambda I-A_{b}\right) x_{b}+B_{b} P_{a}(\lambda) u-B_{b} C_{a}\left(\lambda I-A_{a}\right)^{-1} z_{a}
$$

Based on Lemma 2.6 and [39, Lemma 8.2.8], Assumption 2.8(ii),(iv) imply

$$
X_{b} \subset R\left(\lambda I-A_{b}\right)+\mathcal{R}\left(B_{b} P_{a}(\lambda)\right),
$$

thus there exists $x_{b}$, hence $x$ and $u$, such that (45a) holds. Now (39) holds, i.e., the pair $(A, B)$ is exponentially stabilizable.

\section{Robust output regulation}

The output tracking goal (9) is in the case of abstract linear systems covered by the robust output regulation problem. We start by coupling an error feedback controller with the cascade system (35). The resulting system is called the closed-loop system. We then present the robust output regulation problem, which describes requirements for choosing the controller operators. Finally, we design an error feedback controller, introduced in [34], to solve the robust output regulation problem for the room model.

An error feedback controller on a Hilbert space $Z$ is given by

$$
\begin{aligned}
\dot{z}(t) & =\mathcal{G}_{1} z(t)+\mathcal{G}_{2} e(t), \quad z(0)=z_{0} \in Z, \\
u(t) & =K z(t),
\end{aligned}
$$


where $\mathcal{G}_{1}$ generates a strongly continuous semigroup on $Z, \mathcal{G}_{2} \in \mathcal{L}(Y, Z), K \in$ $\mathcal{L}(Z, U)$ and $e(t)=y(t)-y_{\text {ref }}(t)$ is the regulation error. Coupling the controller with the cascade system (35) yields the closed-loop system, see [23,35],

$$
\begin{aligned}
\dot{x}_{e}(t) & =A_{e} x_{e}(t)+B_{e} w_{\mathrm{ext}}(t), \quad x_{e}(0)=x_{e}, \\
e(t) & =C_{e} x_{e}(t)+D_{e} w_{\mathrm{ext}}(t)
\end{aligned}
$$

on the Hilbert space $X_{e}:=X \times Z$ with the state $x_{e}=[x, z]^{\mathrm{T}}$. Here, $w_{\mathrm{ext}}=\left[u_{d}, y_{\mathrm{ref}}\right]^{\mathrm{T}}$,

$$
\begin{aligned}
A_{e} & =\left[\begin{array}{cc}
A & B K \\
\mathcal{G}_{2} C & \mathcal{G}_{1}
\end{array}\right], & B_{e} & =\left[\begin{array}{cc}
B_{d} & 0 \\
\mathcal{G}_{2} D_{d}-\mathcal{G}_{2}
\end{array}\right], \\
C_{e} & =[C, 0], & D_{e} & =\left[D_{d},-I\right] .
\end{aligned}
$$

The robust output regulation problem Design a controller $\left(\mathcal{G}_{1}, \mathcal{G}_{2}, K\right)$ such that the following hold:

(I) The closed-loop system is exponentially stable.

(II) There exist $M_{r}, \omega_{r}>0$ such that for all initial states $x_{e 0} \in X_{e}$ of the closed-loop system and for all reference signals $y_{\text {ref }}$ in (2) and disturbance signals $u_{d}$ in (3)

$$
\left\|y(t)-y_{\mathrm{ref}}(t)\right\| \leq M_{r} \mathrm{e}^{-\omega_{r} t}\left(\left\|x_{e 0}\right\|+\|\Lambda\|\right)
$$

where $\Lambda$ is a vector consisting of the coefficients of the polynomials $a_{i}(t), b_{i}(t)$, $c_{i}(t)$ and $d_{i}(t)$ of $y_{\text {ref }}$ and $u_{d}$.

(III) If $A, B, B_{d}, C, D_{d}$ in (35) are perturbed to $\tilde{A}, \tilde{B}, \tilde{B}_{d}, \tilde{C}, \tilde{D}_{d}$ in such a way that the closed-loop system remains exponentially stable, then for all $x_{e 0} \in X_{e}$ and for all signals of the form (2), (3) the regulation error satisfies (51) for some $\tilde{M}_{r}, \tilde{\omega}_{r}>0$.

By the internal model principle, an error feedback controller solves the robust output regulation problem precisely when the closed-loop system is exponentially stable and the controller incorporates a suitable internal model of the reference and disturbance signals [35]. That is, the controller must include the dynamics of the reference signal (2) and the disturbance signal (3) reduplicated according to the dimension $p_{Y}$ of the output space $Y$, cf. the first step of the controller design algorithm. The following lemma, i.e., $(A, B, C)$ having no transmission zeros at the relevant frequencies, is a standard necessary property for solvability of the robust output regulation problem.

Assumption 3.1 None of the systems $\left(A_{b}, B_{b}, C_{b}\right),\left(A_{a}, B_{a}, C_{a}\right)$ and $\left(A_{s}, B_{s}, C_{s}\right)$ has transmission zeros at the frequencies $\left\{i \omega_{k}\right\}_{k=0}^{q_{s}}$, i.e., for bounded stabilizing feedback operators $K_{b}, K_{a}$ and $K_{s}$ the transfer functions $P_{b, K_{b}}\left(i \omega_{k}\right)=C_{b}\left(i \omega_{k} I-\right.$ $\left.A_{b}-B_{b} K_{b}\right)^{-1} B_{b}, P_{a, K_{a}}\left(i \omega_{k}\right)=C_{a}\left(i \omega_{k} I-A_{a}-B_{a} K_{a}\right)^{-1} B_{a}$ and $P_{s, K_{s}}\left(i \omega_{k}\right)=$ $C_{s}\left(i \omega_{k} I-A_{s}-B_{s} K_{s}\right)^{-1} B_{s}$ are surjective for $k=0,1, \ldots, q_{s}$.

Lemma 3.2 Given Assumption 3.1, the cascade system $(A, B, C)$ has no transmission zeros at the frequencies $\left\{i \omega_{k}\right\}_{k=0}^{q_{s}}$. 
The proof follows immediately since the transfer function of the cascade system is product of the transfer functions of the three subsystems.

The particular controller design we propose for the cascade system is the one in [34, Sec. III.A], see also [36] for its boundary control system implementation.

I The observer-based finite-dimensional controller is given by

$$
\begin{aligned}
\dot{z}_{1}(t) & =G_{1} z_{1}(t)+G_{2} e(t), \\
\dot{z}_{2}(t) & =\left(A_{L}^{r}+B_{L}^{r} K_{2}^{r}\right) z_{2}(t)+B_{L}^{r} K_{1}^{N} z_{1}(t)-L^{r} e(t), \\
u(t) & =K_{1}^{N} z_{1}(t)+K_{2}^{r} z_{2}(t),
\end{aligned}
$$

and is of the form (50) with $z(t):=\left[z_{1}(t), z_{2}(t)\right]^{\mathrm{T}} \in Z:=Z_{i m} \times \mathbb{C}^{r}$,

$$
\mathcal{G}_{1}=\left[\begin{array}{cc}
G_{1} & 0 \\
B_{L}^{r} K_{1}^{N} & A_{L}^{r}+B_{L}^{r} K_{2}^{r}
\end{array}\right], \quad \mathcal{G}_{2}=\left[\begin{array}{c}
G_{2} \\
-L^{r}
\end{array}\right], \quad K=\left[\begin{array}{ll}
K_{1}^{N} & K_{2}^{r}
\end{array}\right] .
$$

For the cascade system (5)-(8), the operators in (52) are chosen according to the following algorithm.

\section{The Internal Model:}

Choose $Z_{i m}=Y^{n_{0}} \times Y^{2 n_{1}} \times \cdots \times Y^{2 n_{q_{s}}}$, where $n_{i}-1, i \in\left\{0,1, \ldots, q_{s}\right\}$ is the highest-order polynomial coefficient of the corresponding frequency $\omega_{i}$ in (2). Set $G_{1}=\operatorname{diag}\left(J_{0}^{Y}, \ldots J_{q_{s}}^{Y}\right) \in \mathcal{L}\left(Z_{i m}\right)$ and $G_{2}=\left(G_{2}^{k}\right)_{k=0}^{q_{s}} \in \mathcal{L}\left(Y, Z_{i m}\right)$, where

$$
J_{0}^{Y}=\left[\begin{array}{cccc}
0_{p} & I_{p} & & \\
& 0_{p} & \ddots & \\
& & \ddots & I_{p} \\
& & & 0_{p}
\end{array}\right], \quad G_{2}^{0}=\left[\begin{array}{c}
0_{p} \\
\vdots \\
0_{p} \\
I_{p}
\end{array}\right]
$$

and for $k=1 \ldots q_{s}$

$$
J_{k}^{Y}=\left[\begin{array}{cccc}
\Omega_{k} & I_{2 p} & & \\
& \Omega_{k} & \ddots & \\
& & \ddots & I_{2 p} \\
& & & \Omega_{k}
\end{array}\right], \quad G_{2}^{k}=\left[\begin{array}{c}
0_{2 p} \\
\vdots \\
0_{2 p} \\
I_{p} \\
0_{p}
\end{array}\right], \quad \Omega_{k}=\left[\begin{array}{cc}
0_{p} & \omega_{k} I_{p} \\
-\omega_{k} I_{p} & 0_{p}
\end{array}\right]
$$

\section{Plant approximation and stabilization}

For a sufficiently large $N \in \mathbb{N}$, discretize the operators $A_{b}, B_{b}$ and $C_{b}$ to obtain the finite-dimensional approximative system $\left(A_{b}^{N}, B_{b}^{N}, C_{b}^{N}\right)$ on $H_{b}^{N}$. The chosen approximation method should satisfy the following assumption.

Assumption 3.3 The finite-dimensional approximating subspaces $H_{b}^{N}$ of $H_{b}$ have the following property: For any $x_{b} \in H_{b}$ there exists a sequence $x_{b}^{N} \in H_{b}^{N}$ such that

$$
\left\|x_{b}^{N}-x_{b}\right\|_{H_{b}} \rightarrow 0 \text { as } N \rightarrow \infty .
$$


More specifically, the approximation of the cascade system should have the property equivalent to (53), and Assumption 3.3 implies the approximation property for the cascade system.

Lemma 3.4 Let $H^{N}:=H_{b}^{N} \times \mathbb{R}^{n_{a}} \times \mathbb{R}^{n_{s}}$ denote the finite-dimensional approximating subspaces of $H$. Given Assumption 3.3, there exists a sequence $x^{N} \in H^{N}$ such that $\left\|x^{N}-x\right\|_{H} \rightarrow 0$ as $N \rightarrow \infty$.

The proof follows immediately by choosing $x^{N}=\left[x_{b}^{N}, x_{a}, x_{s}\right]^{\mathrm{T}}$, where $x_{b}^{N}$ is such that Assumption 3.3 holds.

Denote the cascade system approximation on $H^{N}$ by $\left(A^{N}, B^{N}, C^{N}\right)$. Let $\alpha_{1}, \alpha_{2} \geq$ 0 and choose $Q_{1} \in \mathcal{L}\left(U_{0}, X\right), Q_{2} \in \mathcal{L}\left(X, Y_{0}\right)$ such that $\left(A+\alpha_{1} I, Q_{1}, C\right)$ and $\left(A+\alpha_{2} I, B, Q_{2}\right)$ are exponentially stabilizable and detectable, where $U_{0}$ and $Y_{0}$ are Hilbert spaces. Denote by $Q_{1}^{N}$ and $Q_{2}^{N}$ the approximations of $Q_{1}$ and $Q_{2}$ on $H^{N}$. Choose $0<R_{1} \in \mathcal{L}(Y)$ and $0<R_{2} \in \mathcal{L}(U)$, and choose $Q_{0} \in \mathcal{L}\left(Z_{i m}, \mathbb{C}^{p_{0}}\right)$ such that $\left(Q_{0}, G_{1}\right)$ is observable. Let

$$
A_{c}^{N}=\left[\begin{array}{cc}
G_{1} & G_{2} C^{N} \\
0 & A^{N}
\end{array}\right], \quad B_{c}^{N}=\left[\begin{array}{c}
0 \\
B^{N}
\end{array}\right], \quad Q_{c}^{N}=\left[\begin{array}{cc}
Q_{0} & 0 \\
0 & Q_{2}^{N}
\end{array}\right]
$$

and solve the finite-dimensional Riccati equations

$$
\begin{aligned}
& \left(A^{N}+\alpha_{1} I\right) \Sigma_{N}+\Sigma_{N}\left(A^{N}+\alpha_{1} I\right)^{*}-\Sigma_{N}\left(C^{N}\right)^{*} R_{1}^{-1} C^{N} \Sigma_{N}=-Q_{1}^{N}\left(Q_{1}^{N}\right)^{*}, \\
& \left(A_{c}^{N}+\alpha_{2} I\right)^{*} \Pi_{N}+\Pi_{N}\left(A_{c}^{N}+\alpha_{2} I\right)-\Pi_{N} B_{c}^{N} R_{2}^{-1}\left(B_{c}^{N}\right)^{*} \Pi_{N}=-\left(Q_{c}^{N}\right)^{*} Q_{c}^{N} .
\end{aligned}
$$

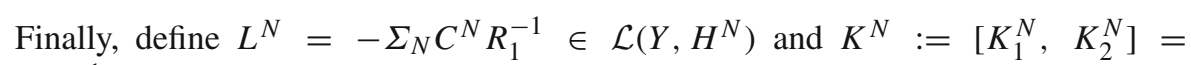
$-R_{2}^{-1}\left(B_{c}^{N}\right)^{*} \Pi_{N} \in \mathcal{L}\left(Z_{i m} \times H^{N}, U\right)$.

\section{Model reduction}

For a sufficiently large $r \leq N$, apply balanced truncation, see [34, Sec. II-B] and the references therein, on the stable system

$$
\left(A^{N}+L^{N} C^{N},\left[B^{N}, L^{N}\right], K_{2}^{N}\right)
$$

to obtain the reduced-order system

$$
\left(A_{L}^{r},\left[B_{L}^{r}, L^{r}\right], K_{2}^{r}\right)
$$

By [34, Thm. III.1], the observer-based finite-dimensional controller solves the robust output regulation problem for a class of systems including the infinitedimensional cascade system (5)-(8). Thus, the following holds for robust output tracking of the linearized translated Boussinesq equations (5) with the observation (12), the actuator dynamics (6) and the sensor dynamics (8).

Theorem 3.5 Assume that the control and disturbance shape functions $\left[b_{v_{I}}, b_{d v_{I}}\right] \in\left(L^{2}\left(\Gamma_{I}\right)\right)^{i},\left[b_{\theta_{I}}, b_{d \theta_{I}}\right] \in\left(L^{2}\left(\Gamma_{I}\right)\right)^{j}$ and $\left[b_{\theta_{H}}, b_{d \theta_{H}}\right] \in\left(L^{2}\left(\Gamma_{H}\right)\right)^{k}$, where $i, j$ and $k$ equal the sum of the number of control and disturbance inputs for 
the inlet velocity, the inlet temperature and the heating strip temperature, respectively. Assume that the observation on the fluid satisfies Assumption 2.1 and the linearized translated Boussinesq equations (5) with the observation (12), the actuator (6) and the sensor (8) satisfy Assumptions 2.7, 2.8 and 3.1. Then, the finite-dimensional low-order controller (52) solves the robust output regulation problem for the cascade system (5)(8), thus achieving the output convergence (9), provided that Assumption 3.3 holds for the approximation method used in the controller design and the orders of approximation $N$ and $r \leq N$ are large enough.

\section{Output tracking example for the room model}

We consider robust output tracking of the linearized translated Boussinesq equations (5) with the actuator dynamics (6) and the sensor dynamics (8) in the rectangular room $\Omega=[0,1] \times[0,1]$ with the inlet, the outlet and the heating strip given by

$$
\begin{aligned}
\Gamma_{I} & =\left\{\xi_{1}=0, \frac{5}{8} \leq \xi_{2} \leq \frac{7}{8}\right\}, \quad \Gamma_{O}=\left\{\xi_{1}=1, \frac{1}{8} \leq \xi_{2} \leq \frac{1}{2}\right\}, \\
\Gamma_{H} & =\left\{\frac{3}{8} \leq \xi_{1} \leq \frac{5}{8}, \xi_{2}=0\right\},
\end{aligned}
$$

which roughly corresponds to Fig. 1. Physical parameters for the Boussinesq equations are chosen as $R e=100, G r=\frac{R e^{2}}{0.9}$ and $\operatorname{Pr}=0.7$. There are three control inputs on the fluid; one on each of $v_{\xi_{1}}$ and $\theta$ within the inlet and one on $\theta$ within the heating strip, with coefficients $\alpha_{v}=\alpha_{\theta}=1$ indicating Robin boundary conditions within the inlet. Additionally, we assume that a single disturbance signal acts within the inlet on $v_{\xi_{2}}$. Now $u_{b}(t)=\left[u_{v}(t), u_{\theta_{I}}(t), u_{\theta_{H}}(t)\right]^{\mathrm{T}} \in \mathbb{R}^{3}$ and $u_{d}(t)=u_{d v_{I}}(t) \in \mathbb{R}$. The control and disturbance shapes are given by

$$
\begin{aligned}
& b_{v_{I}}\left(\xi_{2}\right)=\left[\left.\exp \left(\frac{-0.00004}{\left(\left(5 / 8-\xi_{2}\right)\left(7 / 8-\xi_{2}\right)\right)^{2}}\right)\right|_{\Gamma_{I}}, 0\right]^{\mathrm{T}}, \\
& b_{\theta_{I}}\left(\xi_{2}\right)=\left.\exp \left(\frac{-0.00002}{\left(\left(5 / 8-\xi_{2}\right)\left(7 / 8-\xi_{2}\right)\right)^{2}}\right)\right|_{\Gamma_{I}}, \\
& b_{\theta_{H}}\left(\xi_{1}\right)=\left.\exp \left(\frac{-0.00001}{\left(\left(3 / 8-\xi_{1}\right)\left(5 / 8-\xi_{1}\right)\right)^{2}}\right)\right|_{\Gamma_{H}}, \\
& b_{d v_{I}}\left(\xi_{2}\right)=\left[0,\left.\exp \left(\frac{-0.0003}{\left(\left(5 / 8-\xi_{2}\right)\left(7 / 8-\xi_{2}\right)\right)^{2}}\right)\right|_{\Gamma_{I}}\right]^{\mathrm{T}}
\end{aligned}
$$

with the nonzero components depicted in Fig. 3.

We consider three observations on the linearized Boussinesq equations (5). These are given by

$$
y_{b 1}(t)=\frac{1}{\left|\Omega_{\theta}\right|} \int_{\Omega_{\theta}} \theta(\xi, t) d \xi, \quad y_{b 2}(t)=\frac{1}{\left|\Gamma_{O}\right|} \int_{\Gamma_{O}} \theta(\xi, t) d \xi_{2},
$$



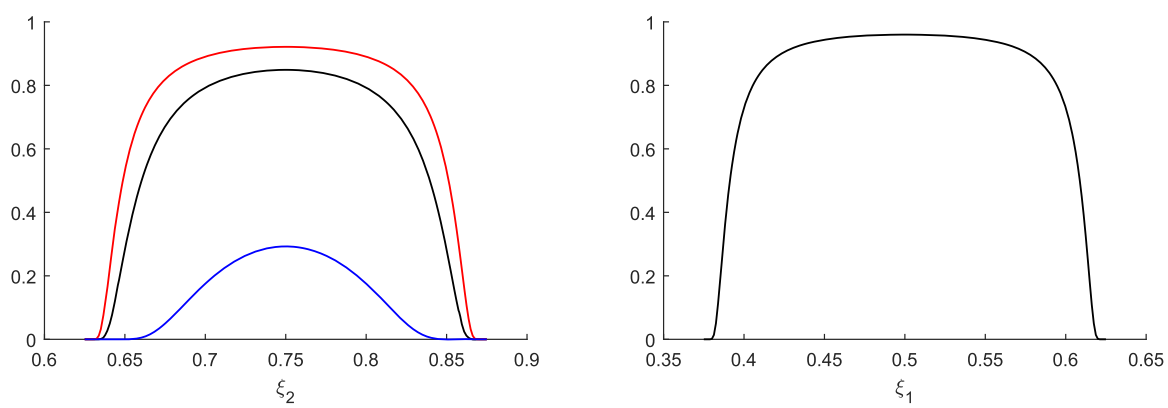

Fig. 3 Control and disturbance shape functions. On the left $b_{v}$ (black), $b_{\theta_{I}}$ (red) and $b_{d v}$ (blue), and on the right $b_{\theta_{H}}$

$$
y_{b 3}(t)=\frac{1}{\left|\Omega_{v}\right|} \int_{\Omega_{v}} v_{\xi_{1}}(\xi, t) d \xi,
$$

where $\Omega_{\theta}=\left[\frac{1}{8}, \frac{2}{8}\right] \times\left[\frac{5}{8}, \frac{6}{8}\right]$ and $\Omega_{v}=\left[\frac{3}{8}, \frac{4}{8}\right] \times\left[\frac{2}{8}, \frac{3}{8}\right]$, and we set $y_{b}=$ $\left[y_{b 1}, y_{b 2}, y_{b 3}\right]^{\mathrm{T}}$. The actuator (6) and the sensor (8) are characterized by the simple choices

$$
A_{a}=A_{s}=-I_{3}, \quad B_{a}=C_{a}=B_{s}=C_{s}=I_{3},
$$

and the reference signals to be tracked are

$$
\begin{aligned}
& y_{\text {ref } 1}(t)=-1+\sin (t)+0.3 \cos (2 t), \quad y_{\text {ref } 2}(t)=0.5 \cos (0.5 t), \\
& y_{\text {ref3 }}(t)=1+0.5 \sin (2 t),
\end{aligned}
$$

respectively. Finally, the disturbance signal is given by $u_{d}(t)=2 \sin (0.5 t)$.

For the simulations, we use a uniform triangulation of $\Omega$ together with the TaylorHood finite element spatial discretization for the Navier-Stokes part of the Boussinesq equations and quadratic elements with the same triangulation for the advectiondiffusion part. The incompressibility condition $\nabla \cdot v=0$ is relaxed by using a penalty method to decouple the pressure term from the velocity, see, e.g., [20, Ch. 5.2] or [24], with the penalty parameter $\epsilon_{p}=10^{-5}$. To approximate the infinite-dimensional system $\left(A_{b}, B_{b}, C_{b}\right)$, we use the mesh size $h_{\text {inf }}=1 / 24$, which results in approximation order $N_{\text {inf }}=6728$ for the system $(A, B, C)$ after accounting for the boundary conditions.

We use Newton's method to calculate a steady-state solution for the Boussinesq equations (4) subject to

$$
\begin{aligned}
& f_{T}(\xi)=5 \sin \left(2 \pi \xi_{1}\right) \cos \left(2 \pi \xi_{2}\right), \\
& f_{w}(\xi)=4\left[\sin \left(2 \pi \xi_{1}\right) \cos \left(2 \pi \xi_{2}\right),-\cos \left(2 \pi \xi_{1}\right) \sin \left(2 \pi \xi_{2}\right)\right]^{\mathrm{T}} .
\end{aligned}
$$

The steady-state solution may be, and according to numerical tests is, non-unique, and we choose the steady state $\left(w_{s s}, T_{s S}\right)$ corresponding to the initial guess given by the 


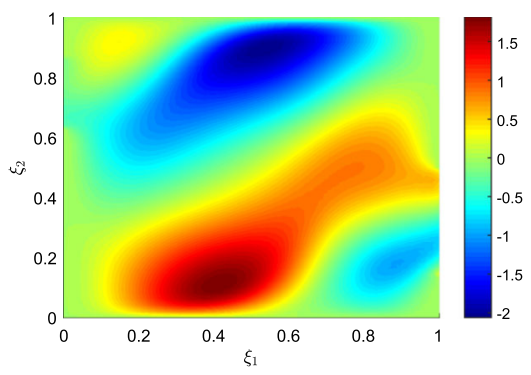

(a) The steady state velocity $w_{s s_{1}}$

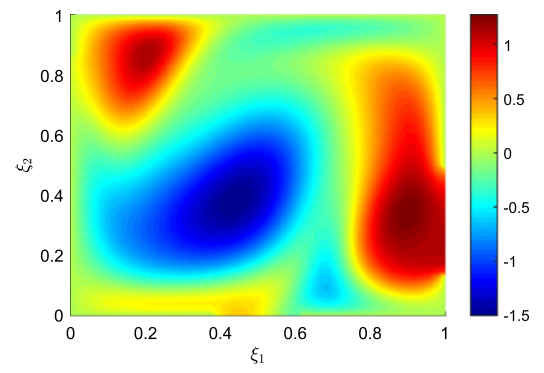

(c) The steady state temperature $T_{s s}$

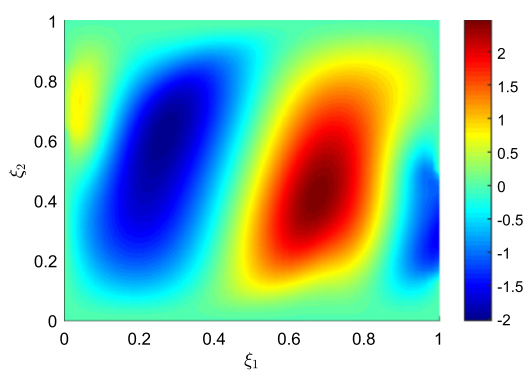

(b) The steady state velocity $w_{s s_{2}}$

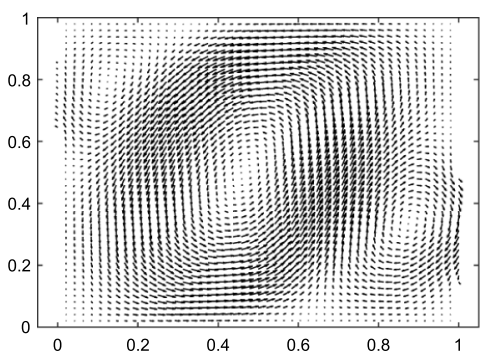

(d) The steady state velocity field $w_{s s}$

Fig. 4 A steady-state solution $\left(w_{s s_{1}}, w_{s s_{2}}, T_{s s}\right)$ of the Boussinesq equations (4)

steady-state solution $\left(w_{i}, T_{i}\right)$ of (4) subject to

$$
\begin{aligned}
& f_{T_{i}}(\xi)=4 \sin \left(2 \pi \xi_{1}\right) \cos \left(2 \pi \xi_{2}\right), \\
& f_{w_{i}}(\xi)=2\left[\sin \left(2 \pi \xi_{1}\right) \cos \left(2 \pi \xi_{2}\right),-\cos \left(2 \pi \xi_{1}\right) \sin \left(2 \pi \xi_{2}\right)\right]^{\mathrm{T}} .
\end{aligned}
$$

The steady-state solution is depicted in Fig. 4.

We observe numerically that for the calculated steady-state solution $A_{b}$, thus also $A$ due to the block triangular structure if rearranged according to the state $\left(x_{a}, x_{b}, x_{s}\right)$, has a single pair of unstable eigenvalues

$$
\lambda_{ \pm} \approx 0.0621 \pm 0.4908 i
$$

Exponential stabilizability, exponential detectability and Assumption 3.1 are checked numerically for the system $\left(A_{b}, B_{b}, C_{b}\right)$, and for the choice (54) they are transferred to the system $(A, B, C)$ by Lemmata 2.9, 2.10 and 3.2.

For the controller construction, we use a coarser linearized Boussinesq equations approximation with the mesh size $h=1 / 16$. Using the penalty method would introduce additional modeling error, so we base the plant approximation $\left(A^{N}, B^{N}, C^{N}\right)$ on the discretized Leray projector instead, see $[7,8,25]$. The discretized Leray projector is used merely as a theoretical tool, and the Riccati equations together with the 


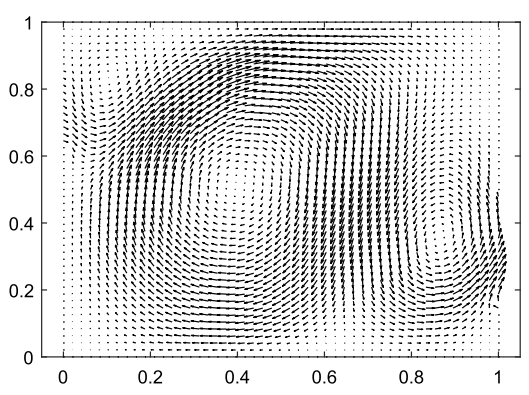

(a) $v_{0}$

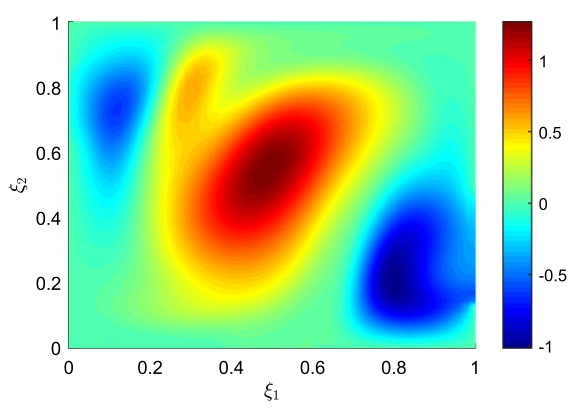

(b) $\theta_{0}$

Fig. 5 The initial state $\left(v_{0}, \theta_{0}\right)$

model-reduced operators $A_{L}^{r}, B_{L}^{r}, K_{2}^{r}, L^{r}$ are instead solved using the differentialalgebraic equation form of the Taylor-Hood-quadratic discretized cascade system. More specifically, we obtain solutions of the Riccati equations using the Generalized Low-Rank Cholesky Factor Newton Method, see Algorithm 2 in [7], with the initial stabilizing feedback solved by the Matlab's icare function using the penalized Taylor-Hood-quadratic discretization and the ADI shift parameters solved using the LYAPACK toolbox for Matlab. Finally, balred function of Matlab is utilized for the order reduction. Parameters of the Riccati equations are chosen as

$$
\alpha_{1}=0.3, \quad \alpha_{2}=0.2, \quad R_{1}=R_{2}=I_{3}, \quad Q_{1} Q_{1}^{*}=I_{X}, \quad Q_{c}^{*} Q_{c}=I_{Z_{i m} \times X} .
$$

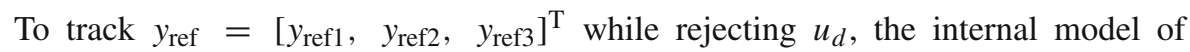
the controller has 4 frequencies. Due to $y_{\text {ref }}$ and $u_{d}$ having constant coefficients, $\operatorname{dim} Z_{i m}=3+3 \cdot 3 \cdot 2=21$, thus us using the reduction order $r=20$ results in the controller order $\operatorname{dim} Z=\operatorname{dim} Z_{\text {im }}+r=41$.

Remark 4.1 Assumption 3.3 is not satisfied by the mixed finite element methods such as the Taylor-Hood discretization implemented in this work. Convergence properties of the approximation-based controllers for mixed finite elements are studied in [9], but the presented results are incomplete for our use. As such, finding suitable approximation methods or verifying convergence properties of commonly used approximation methods, such as mixed finite elements, requires further work.

For the simulations, we choose as the initial state of the closed-loop system

$$
\begin{aligned}
& {\left[v_{0}, \theta_{0}, x_{a 0}, x_{s 0}, z_{0}\right]^{\mathrm{T}}} \\
& =\left[w_{i}-w_{s s}, T_{i}-T_{s s}, 0,0,0\right]^{\mathrm{T}} \in X_{v} \times L^{2}(\Omega) \times \mathbb{R}^{3} \times \mathbb{R}^{3} \times Z
\end{aligned}
$$

and the state components $v_{0}$ and $\theta_{0}$ are shown in Fig. 5.

Output tracking performance of the controller for $t \in[0,50]$ is illustrated in Fig. 6 .

The system output converges to the reference output with accurate tracking for $t>30$. Initial oscillations of the observation are reasonable, but the state $(v, \theta)$ of the 


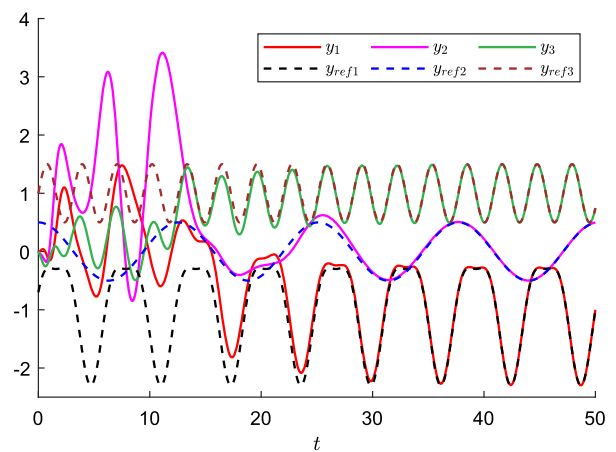

Fig. 6 The system output $y=\left[\begin{array}{lll}y_{1}, & y_{2}, y_{3}\end{array}\right]^{\mathrm{T}}$ and the reference output $y_{\text {ref }}=\left[\begin{array}{ll}y_{\text {ref1 } 1}, & y_{\text {ref2 } 2}, y_{\text {ref } 3}\end{array}\right]^{\mathrm{T}}$

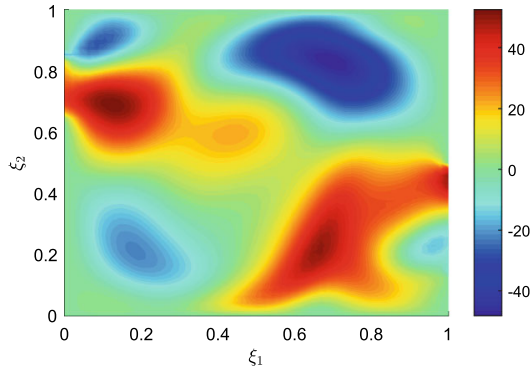

(a) The velocity $v_{1}(\xi, 50)$

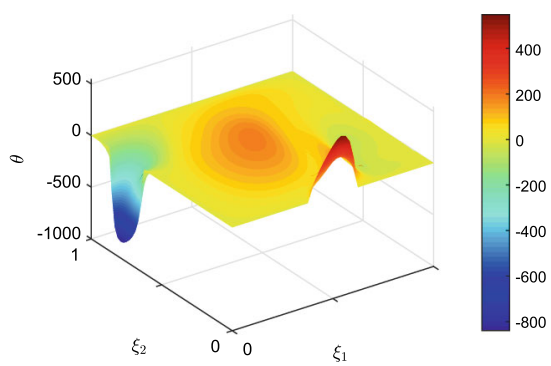

(c) The temperature $\theta(\xi, 50)$

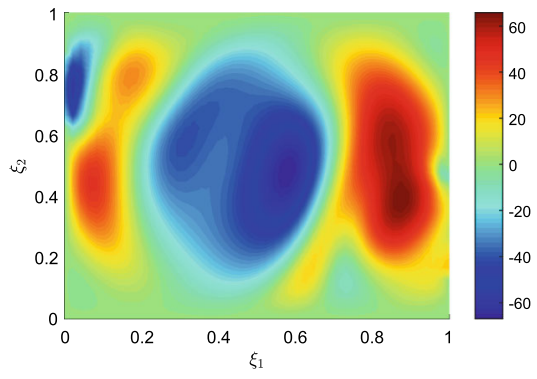

(b) The velocity $v_{2}(\xi, 50)$

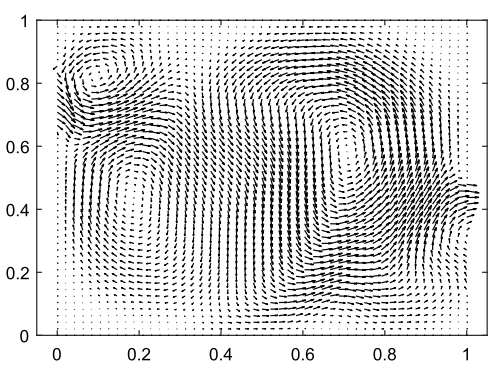

(d) The velocity field $v(\xi, 50)$

Fig. 7 State $\left(v_{1}, v_{2}, \theta\right)$ of the linearized translated Boussinesq equations at the time $t=50$

linearized translated Boussinesq equations experiences significant oscillations. The temperature values near the controlled strips $\Gamma_{I}$ and $\Gamma_{H}$ are a prime example of this behavior, as Fig. 7 suggests, while incompressibility leads to "less localized" velocity states.

The controlled strips actually maintain large amplitudes for both the velocity $v$ and the temperature $\theta$ throughout the simulation disregarding the initial transient, as evident from Fig. 8. 


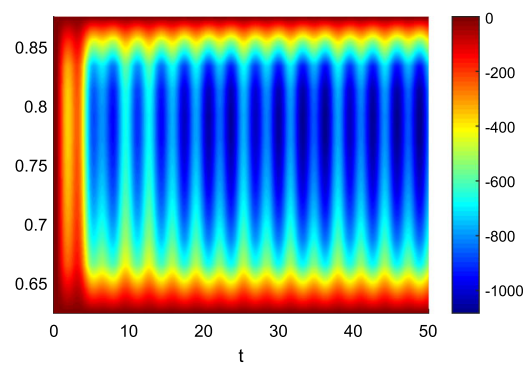

(a) The temperature profile within $\Gamma_{I}$

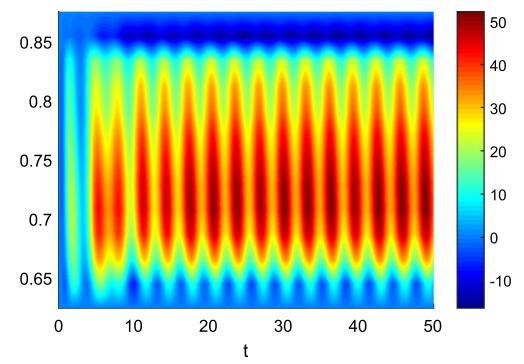

(c) The $\xi_{1}$-velocity profile within $\Gamma_{I}$

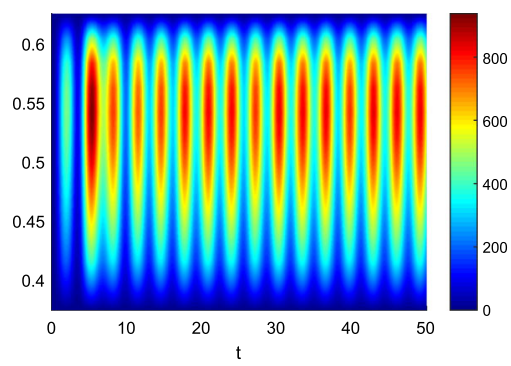

(b) The temperature profile within $\Gamma_{H}$

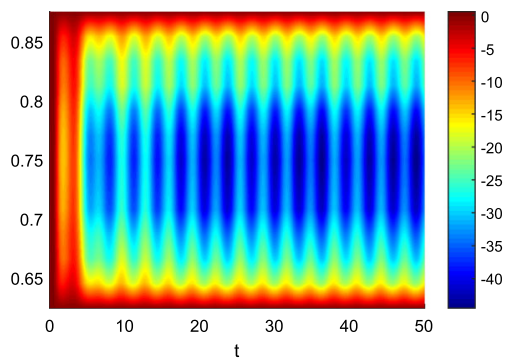

(d) The $\xi_{2}$-velocity profile within $\Gamma_{I}$

Fig. 8 State components within the controlled strips for $t \in[0,50]$

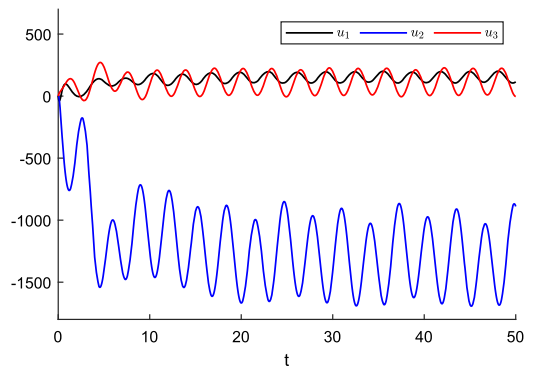

(a) The plant input $u=\left[u_{1}, u_{2}, u_{3}\right]^{T}$

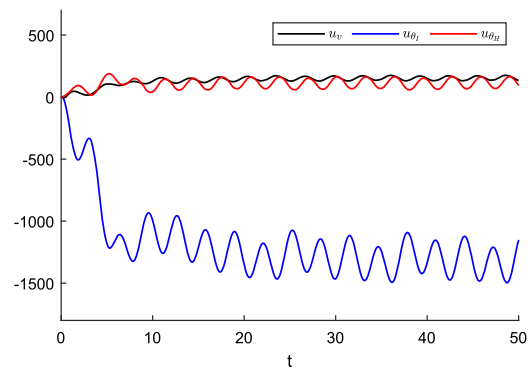

(b) The boundary input $u_{b}=\left[u_{v}, u_{\theta_{I}}, u_{\theta_{H}}\right]^{T}$

Fig. 9 Plant input $u$ and the corresponding boundary input $u_{b}$ generated by the actuator

Similarly, the boundary inputs to the room do not change sign after the transient, see Fig. 9, although the plant input component $u_{3}$ just barely does. 


\section{Conclusion}

We studied robust temperature and velocity output tracking of a two-dimensional room model with the fluid dynamics governed by the linearized Boussinesq equations. For the room model, we considered the natural case of the control applied on the fluid via the boundary and the observations performed on the fluid at the boundary. Related to the control and the observation operations, we modeled actuator and sensor dynamics of the system, thus the complete room model was an ODE-PDE-ODE cascade. We examined effects of the added actuator and sensor dynamics on the system properties such as semigroup generation, exponential stabilizability and exponential detectability, and implemented an internal model-based error feedback controller design for robust temperature and velocity output tracking for the room model. In addition to being robust, the controller is suitable for unstable systems, requires only output information instead of full state information and is of low order for efficient applicability.

As an example, we illustrated robust output tracking of the linearized Boussinesq equations with actuator and sensor dynamics in the case of three boundary controls, a mix of one boundary and two in-domain observations and a boundary disturbance, each affecting either a velocity or the temperature component of the system. The controller achieved accurate tracking with relatively small transient observation oscillation, although the system state reaches large absolute values locally. Analogous approach of actuator and sensor modeling can be applied for robust output tracking of a class of linear systems with boundary control and observation.

Acknowledgements The authors would like to thank the anonymous reviewers as well as Jukka-Pekka Humaloja for providing helpful feedback on the original manuscript.

Open Access This article is licensed under a Creative Commons Attribution 4.0 International License, which permits use, sharing, adaptation, distribution and reproduction in any medium or format, as long as you give appropriate credit to the original author(s) and the source, provide a link to the Creative Commons licence, and indicate if changes were made. The images or other third party material in this article are included in the article's Creative Commons licence, unless indicated otherwise in a credit line to the material. If material is not included in the article's Creative Commons licence and your intended use is not permitted by statutory regulation or exceeds the permitted use, you will need to obtain permission directly from the copyright holder. To view a copy of this licence, visit http://creativecommons.org/licenses/by/4.0/.

\section{References}

1. Aulisa E, Gilliam D (2016) A practical guide to geometric regulation for distributed parameter systems. Monographs and research notes in mathematics. CRC Press, Boca Raton

2. Aulisa E, Gilliam DS, Pathiranage TW (2018) Analysis of an iterative scheme for approximate regulation for nonlinear systems. Int J Robust Nonlinear Control 28(8):3140-3173. https://doi.org/10.1002/ rnc. 4074

3. Aulisa E, Gilliam DS, Pathiranage TW (2019) Analysis of the error in an iterative algorithm for asymptotic regulation of linear distributed parameter control systems. ESAIM Math Model Numer Anal 53(5):1577-1606. https://doi.org/10.1051/m2an/2019021

4. Badra M (2012) Abstract settings for stabilization of nonlinear parabolic system with a Riccati-based strategy. Application to Navier-Stokes and Boussinesq equations with Neumann or Dirichlet control. Discrete Contin Dy. Syst 252(09):5042-5075 (2012). https://doi.org/10.3934/dcds.2012.32.1169 
5. Badra M, Takahashi T (2014) On the Fattorini criterion for approximate controllability and stabilizability of parabolic systems. ESAIM Control Optim. Calc. Var. 20(3):924-956. https://doi.org/10.1051/ cocv/2014002

6. Banks HT, Ito K (1997) Approximation in LQR problems for infinite-dimensional systems with unbounded input operators. J Math Syst Estim Control 7(1):34

7. Bänsch E, Benner P, Saak J, Weichelt HK (2015) Riccati-based boundary feedback stabilization of incompressible Navier-Stokes flows. SIAM J Sci Comput 37(2):A832-A858. https://doi.org/10.1137/ 140980016

8. Benner P, Heiland J (2015) Lqg-balanced truncation low-order controller for stabilization of laminar flows. In: King R (ed) Active Flow Combust Control. Springer, Cham, pp 365-379

9. Benner P, Heiland J (2017) Convergence of approximations to Riccati-based boundary-feedback stabilization of laminar flows. IFAC-PapersOnLine 50(1):12296-12300. https://doi.org/10.1016/j.ifacol. 2017.08.2476

10. Borggaard J, Cliff E, Gugercin S (2012) Model reduction for indoor-air behavior in control design for energy-efficient buildings. In: 2012 American Control Conference (ACC), pp 2283-2288. https://doi. org/10.1109/ACC.2012.6315235

11. Burns JA, Cliff EM (2014) Control of hyperbolic PDE systems with actuator dynamics. In: 53rd IEEE conference on decision and control, pp 2864-2869

12. Burns JA, He X, Hu W (2016) Feedback stabilization of a thermal fluid system with mixed boundary control. Comput Math Appl 71(11):2170-2191. https://doi.org/10.1016/j.camwa.2016.01.011

13. Burns JA, Zietsman L (2012) On the inclusion of actuator dynamics in boundary control of distributed parameter systems. IFAC Proc 45(19):138-142. https://doi.org/10.3182/20120829-3-IT-4022.00039

14. Byrnes CI, Laukó IG, Gilliam DS, Shubov VI (2000) Output regulation for linear distributed parameter systems. IEEE Trans Autom Control 45(12):2236-2252. https://doi.org/10.1109/9.895561

15. Cheng A, Morris K (2003) Well-posedness of boundary control systems. SIAM J Control Optim 42(4):1244-1265. https://doi.org/10.1137/S0363012902384916

16. Curtain RF, Zwart H (1995) An introduction to infinite-dimensional linear systems theory. Texts in applied mathematics, vol 21. Springer, New York. https://doi.org/10.1007/978-1-4612-4224-6

17. Davison E (1976) The robust control of a servomechanism problem for linear time-invariant multivariable systems. IEEE Trans Autom Control 21(1):25-34. https://doi.org/10.1109/TAC.1976.1101137

18. Deutscher J (2011) Output regulation for linear distributed-parameter systems using finite-dimensional dual observers. Autom J IFAC 47(11):2468-2473. https://doi.org/10.1016/j.automatica.2011.08.033

19. Francis B, Wonham W (1975) The internal model principle for linear multivariable regulators. Appl Math Optim 2:170-194. https://doi.org/10.1007/BF01447855

20. Gunzburger M (1989) Finite element methods for viscous incompressible flows: a guide to theory, practice, and algorithms. Academic Press, London

21. Haine G (2017) Closed-loop perturbations of well-posed linear systems. IFAC-PapersOnLine 50(1):4546-4551. https://doi.org/10.1016/j.ifacol.2017.08.729

22. Hämäläinen T, Pohjolainen S (2000) A finite-dimensional robust controller for systems in the CDalgebra. IEEE Trans Autom Control 45(3):421-431. https://doi.org/10.1109/9.847722

23. Hämäläinen T, Pohjolainen S (2010) Robust regulation of distributed parameter systems with infinite-dimensional exosystems. SIAM J Control Optim 48(8):4846-4873. https://doi.org/10.1137/ 090757976

24. He X, Hu W, Zhang Y (2018) Observer-based feedback boundary stabilization of the Navier-Stokes equations. Comput Methods Appl Mech Eng 339:542-566. https://doi.org/10.1016/j.cma.2018.05.008

25. Heinkenschloss M, Sorensen DC, Sun K (2008) Balanced truncation model reduction for a class of descriptor systems with application to the Oseen equations. SIAM J Sci Comput 30(2):1038-1063. https://doi.org/10.1137/070681910

26. Hu W, Singler JR, Zhang Y (2016) Feedback control of a thermal fluid based on a reduced order observer. In: 10th IFAC symposium on nonlinear control systems NOLCOS, pp 116-121 (2016)

27. Huhtala K, Paunonen L, Hu W (2021) Robust output tracking for a room temperature model with distributed control and observation. IFAC-PapersOnLine 54(9):462-467. https://doi.org/10.1016/j.ifacol. 2021.06.103

28. Humaloja JP, Kurula M, Paunonen L (2019) Approximate robust output regulation of boundary control systems. IEEE Trans Autom Control 64(6):2210-2223

29. Kim H (2012) The existence and uniqueness of very weak solutions of the stationary Boussinesq system. Nonlinear Anal 75(1):317-330. https://doi.org/10.1016/j.na.2011.08.035 
30. Maz'ya V, Rossmann J (2009) Mixed boundary value problems for the stationary Navier-Stokes system in polyhedral domains. Arch Rat Mech Anal 194(2):669-712. https://doi.org/10.1007/s00205008-0171-z

31. Morris K (2018) The role of sensor and actuator models in control of distributed parameter systems. Springer, Cham, pp 245-257. https://doi.org/10.1007/978-3-319-67068-3_18

32. Nguyen PA, Raymond JP (2015) Boundary stabilization of the Navier-Stokes equations in the case of mixed boundary conditions. SIAM J Control Optim 53(5):3006-3039. https://doi.org/10.1137/ $13091364 \mathrm{X}$

33. Paunonen L (2016) Controller design for robust output regulation of regular linear systems. IEEE Trans Autom Control 61(10):2974-2986. https://doi.org/10.1109/TAC.2015.2509439

34. Paunonen L, Phan D (2020) Reduced order controller design for robust output regulation. IEEE Trans Autom Control 65(6):2480-2493

35. Paunonen L, Pohjolainen S (2010) Internal model theory for distributed parameter systems. SIAM J Control Optim 48(7):4753-4775. https://doi.org/10.1137/090760957

36. Phan D, Paunonen L (2021) Finite-dimensional controllers for robust regulation of boundary control systems. Math Control Relat Fields 11(1):95-117. https://doi.org/10.3934/mcrf.2020029

37. Ramaswamy M, Raymond JP, Roy A (2019) Boundary feedback stabilization of the Boussinesq system with mixed boundary conditions. J Differ Equ 266(7):4268-4304. https://doi.org/10.1016/j.jde.2018. 09.038

38. Rebarber R, Weiss G (2003) Internal model based tracking and disturbance rejection for stable wellposed systems. Automatica 39(9):1555-1569

39. Staffans O (2005) Well-posed linear systems, Encyclopedia of mathematics and its applications, vol 103. Cambridge University Press, Cambridge. https://doi.org/10.1017/CBO9780511543197

40. Tucsnak M, Weiss G (2009) Observation and control for operator semigroups. Birkhäuser advanced texts. Basel textbooks. Birkhäuser Verlag, Basel

41. Tucsnak M, Weiss G (2014) Well-posed systems - the LTI case and beyond. Autom J IFAC 50(7):17571779. https://doi.org/10.1016/j.automatica.2014.04.016

42. Wang G (2003) Stabilization of the Boussinesq equation via internal feedback controls. Nonlinear Anal 52(2):485-506. https://doi.org/10.1016/S0362-546X(02)00114-1

43. Weiss G (1989) The representation of regular linear systems on Hilbert spaces. Control and estimation of distributed parameter systems (Vorau, 1988). Int Ser Numer Math 91:401-416

44. Weiss G (1994) Regular linear systems with feedback. Math Control Signals Syst 7:23-57. https://doi. org/10.1007/BF01211484

45. Xu X, Dubljevic S (2017) Output and error feedback regulator designs for linear infinite-dimensional systems. Autom J IFAC 83:170-178. https://doi.org/10.1016/j.automatica.2017.06.003

46. Zimmer B, Lipshitz S, Morris K, Vanderkooy J, Obasi E (2003) An improved acoustic model for active noise control in a duct. J Dyn Syst Meas Control. https://doi.org/10.1115/1.1592192

Publisher's Note Springer Nature remains neutral with regard to jurisdictional claims in published maps and institutional affiliations. 